\title{
PRICING DEATH: \\ FRAMEWORKS FOR THE VALUATION AND SECURITIZATION OF MORTALITY RISK*
}

\author{
BY
}

Andrew J.G. Cairns, David Blake and Kevin Dowd

\begin{abstract}
It is now widely accepted that stochastic mortality - the risk that aggregate mortality might differ from that anticipated - is an important risk factor in both life insurance and pensions. As such it affects how fair values, premium rates, and risk reserves are calculated.

This paper makes use of the similarities between the force of mortality and interest rates to examine how we might model mortality risks and price mortality-related instruments using adaptations of the arbitrage-free pricing frameworks that have been developed for interest-rate derivatives. In so doing, the paper pulls together a range of arbitrage-free (or risk-neutral) frameworks for pricing and hedging mortality risk that allow for both interest and mortality factors to be stochastic. The different frameworks that we describe - short-rate models, forward-mortality models, positive-mortality models and mortality market models - are all based on positive-interest-rate modelling frameworks since the force of mortality can be treated in a similar way to the short-term risk-free rate of interest. While much of this paper is a review of the possible frameworks, the key new development is the introduction of mortality market models equivalent to the LIBOR and swap market models in the interest-rate literature.

These frameworks can be applied to a great variety of mortality-related instruments, from vanilla longevity bonds to exotic mortality derivatives.
\end{abstract}

\section{KEYWORDS}

Stochastic mortality, term structure of mortality, survivor index, spot survival probabilities, spot force of mortality, forward mortality surface, short-rate models, forward mortality models, positive mortality framework, mortality market models, annuity market model, SCOR market model.

\footnotetext{
* First version presented at the 14th International AFIR Colloquium, Boston, 2004, under the title Pricing Frameworks for Securitization of Mortality Risk, available online at http://afir2004.soa.org/ afir_papers.htm.
} 


\section{INTRODUCTION}

A large number of products in life insurance and pensions by their very nature have mortality as a primary source of risk. By this we mean that products are exposed to unanticipated changes over time in the mortality rates of the appropriate reference population. For example, annuity providers are exposed to the risk that the mortality rates of pensioners will fall at a faster rate than accounted for in their pricing and reserving calculations, and life insurers are exposed to the risk of unexpected increases in mortality (a recent example being those due to HIV/AIDS). On the asset side of their balance sheets, insurance companies are also exposed to investment risks and, since their investment portfolios are predominantly fixed income, this means that they are heavily exposed to interest-rate risk.

However, there is a huge gap in the tools available to model these two types of risk. On the one hand, the theory and practice of interest-rate modelling is very well developed (see, for example, Vasicek, 1977, Cox, Ingersoll and Ross, 1985, Heath, Jarrow and Morton, 1992, Miltersen, Sandmann and Sondermann, 1997, Brace, Gatarek and Musiela, 1997, Jamshidian, 1997, Brigo and Mercurio, 2001, James and Webber, 2002, Rebonato, 2002, and Cairns, 2004b). On the other hand, the state and practice of mortality risk modelling is relatively primitive.

Previous authors (see, for example, Milevsky and Promislow, 2001, Dahl, 2004, and Biffis, 2005) have observed that there are important similarities between the force of mortality and interest rates. In particular, both are positive processes, have term structures, and are fundamentally stochastic in nature. Milevsky and Promislow (2001) and Dahl (2004) exploited these similarities to model mortality risk using methods developed in interest-rate modelling. In a similar fashion, Biffis (2005) exploited the similarities between mortality risk and credit risk. This paper seeks to develop their insights further. In particular, it makes use of the similarities between mortality and interest rate risks to show how we can model mortality risks and price mortality-related instruments using adaptations of the arbitrage-free pricing frameworks that have been developed for interest-rate derivatives. In so doing, it develops a range of arbitrage-free frameworks for pricing and hedging mortality risk that allow for both interest and mortality factors to be stochastic.

\subsection{Evidence that mortality is stochastic}

To motivate our discussion, we will first summarise some evidence that confirms that mortality improvements are indeed stochastic. We then briefly discuss the state of the art in mortality modelling, and go on to consider some financial instruments whose values depend on mortality and where it is therefore important to model mortality risk factors in an appropriate way.

The idea that mortality is stochastic is not a new one, and it has been evident for many years that mortality rates have been evolving in an apparently 


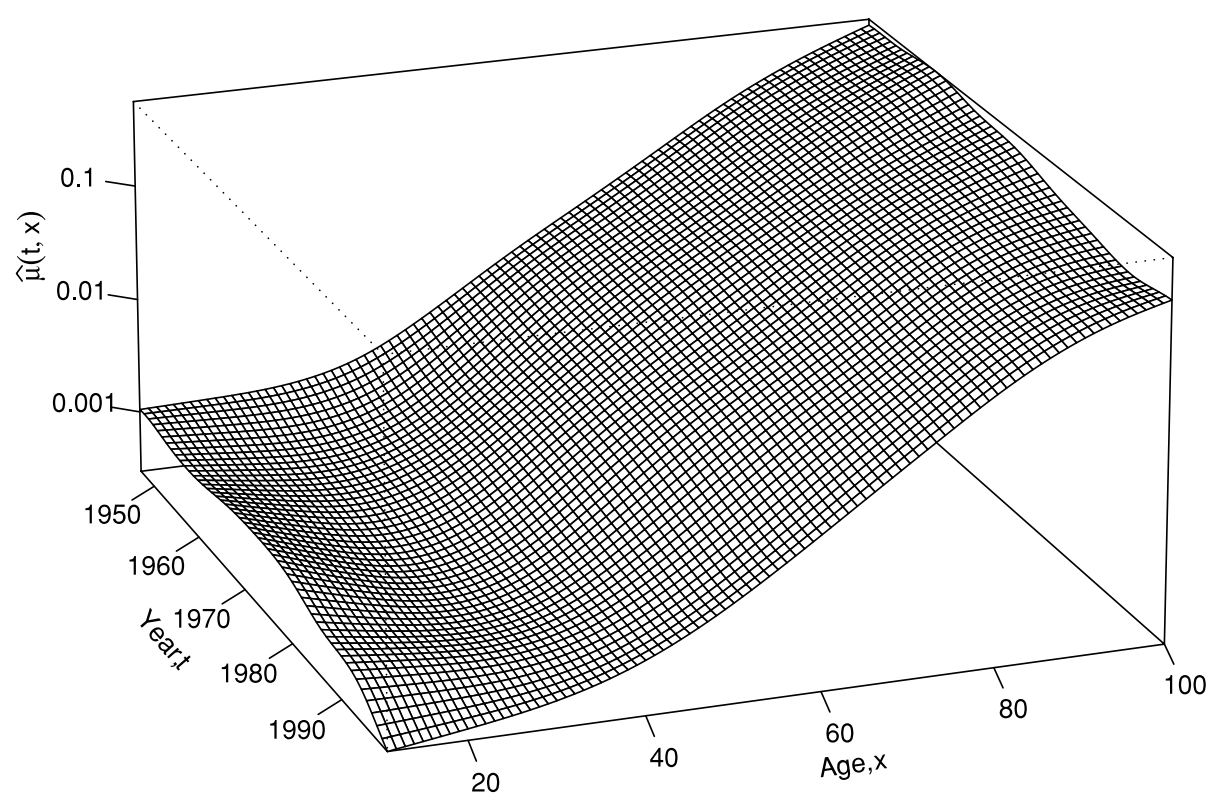

Figure 1.1. Fitted values using P-splines for the force of mortality $\hat{\mu}(t, x)$ for the years $t=1947$ to 1999 and for ages $x=11$ to 100 from Currie, Durban and Eilers, 2004.

(Data: UK males, assured lives).

stochastic fashion. The uncertainty of mortality forecasts is illustrated in recent work by Currie, Durban and Eilers (2004) (hereafter CDE) which analysed historical trends in mortality using P-splines. The fitted surface of values for the force of mortality ${ }^{1} \hat{\mu}(t, x)$ is plotted on a $\log$ scale in Figure 1.1 while the development of the force of mortality for specific ages over time relative to values in 1947 is plotted in Figure 1.2. Figure 1.2 reveals some detail that we cannot easily see in Figure 1.1: specifically that the rate of improvement has varied significantly over time, and that the improvements have varied substantially between different age groups. CDE also constructed confidence bounds for the future development of mortality rates. Inevitably, these confidence bounds get wider as the forecast horizon lengthens and CDE found that even 15-20 years ahead the bounds are very wide. In general terms, the analysis of CDE, as well as other analyses using the stochastic mortality models discussed below, indicates that future mortality improvements cannot be forecast with any degree of precision. Other studies (e.g., Forfar and Smith, 1987, Macdonald et al, 1998, Willets, 1999, and Macdonald et al, 2003) have come to similar conclusions.

1 For an individual aged $x$ at time $t$ and still alive at that time, the force of mortality $\mu(t, x)$ represents the instantaneous death rate: that is, for a small interval of time $d t$, the probability of death between $t$ and $t+d t$ is $\mu(t, x) d t+o(d t)$ as $d t \rightarrow 0$ (that is, approximately $\mu(t, x) d t$ for small $d t$ ). The force of mortality $\mu(t, x)$ is described in more detail at the start of Section 2. 


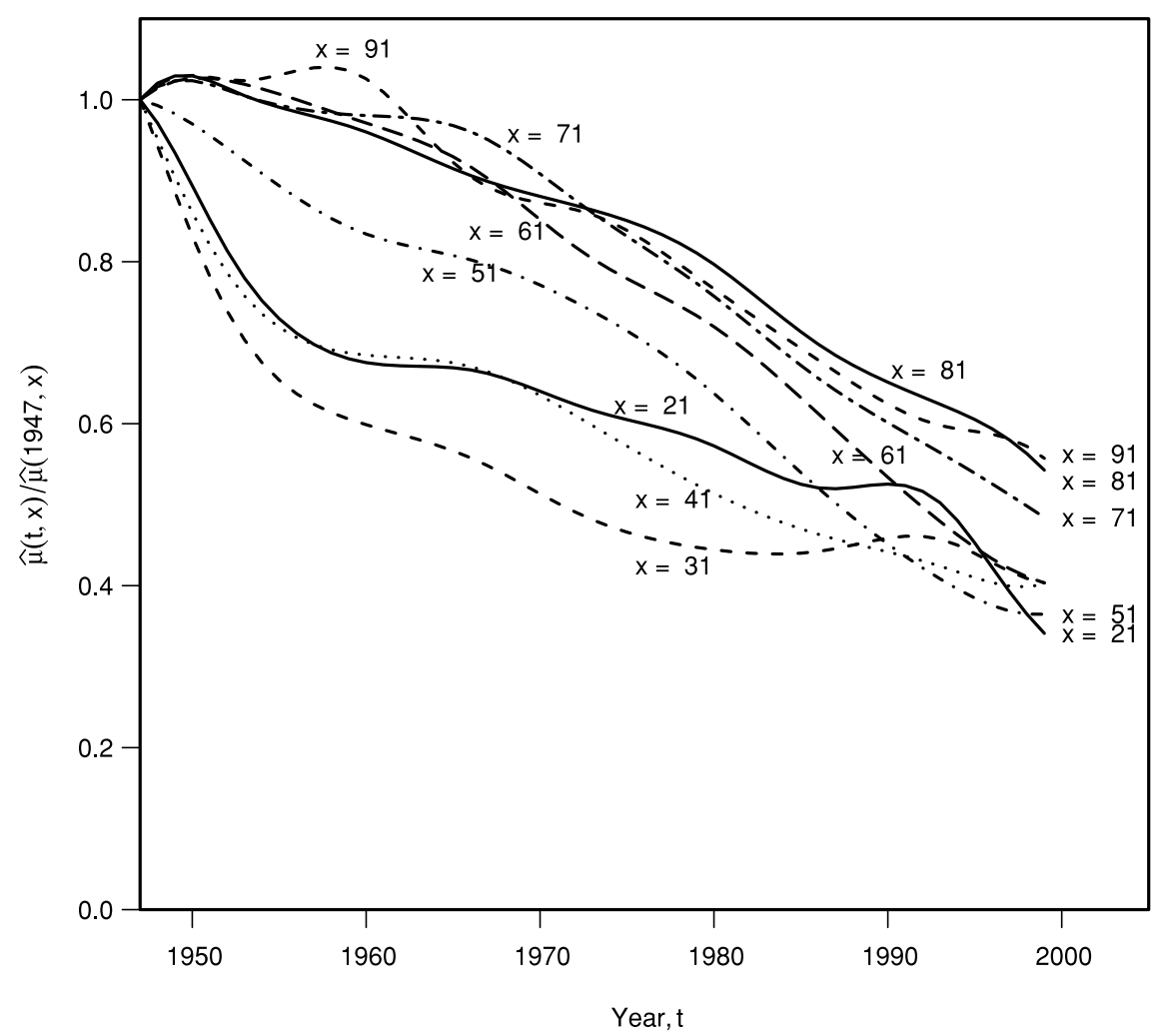

FIGURE 1.2. $\hat{\mu}(t, x) / \hat{\mu}(1947, x)$ : Fitted values using P-splines for the force of mortality $\hat{\mu}(t, x)$, relative to the 1947 value for the years $t=1947$ to 1999 and for ages $x=21,31,41,51,61,71,81$ and 91 from Currie,

Durban and Eilers, 2004. Note that the pattern of improvements is different at different ages. (Data: UK males, assured lives).

We focus in this paper on systematic mortality risk: the risk that aggregate mortality rates differ from those anticipated. Mortality risk can manifest itself in different ways and to help differentiate between them we will employ the following taxonomy. The term mortality risk will be used to cover all forms of deviations in aggregate mortality rates from those anticipated at different ages and over different time horizons. Longevity risk will be used to refer to the risk that, in the long term, aggregate survival rates for identified cohorts are higher than anticipated. Short-term, catastrophic mortality risk will refer to the risk that, over short periods of time, mortality rates are very much higher than would normally be experienced.

\subsection{Recent models of mortality}

A number of recent studies have sought to model mortality as a stochastic process. We shall see presently that all these studies bar one (Lin and Cox, 2005a) can 
be seen as belonging to one of the more general frameworks that we will describe later in this paper. We will describe these briefly here and in the Appendices. The majority of work in this field has concentrated on what we describe as short-rate models for mortality: that is, they are modelling the spot mortality rates $^{2}, q(t, x)$, or the spot force of mortality, $\mu(t, x)$.

The most coherent group of papers that use the short-rate-modelling framework in discrete time build on the original work of Lee and Carter (1992) (see, also, Lee, 2000b). This study introduced a simple model for central mortality rates involving both age-dependent and time-dependent terms and applied it to US population data (see Appendix A for further details). The time-dependency is modelled using a univariate ARIMA time-series model implying that changes in the mortality curve at all ages are perfectly correlated. Brouhns, Denuit and Vermunt (2002) applied the same model to Belgian data and also improved some of the statistical aspects of Lee and Carter's work. The possibility of imperfect mortality correlation was then investigated by Renshaw and Haberman (2003) who extend the Lee and Carter approach by adding a second time-dependent set of changes.

A second approach that also uses the short-rate-modelling framework in discrete time has been proposed by Lee (2000a) and Yang (2001). They take a deterministic projection of the spot mortality rates, $\hat{q}(t, x)$, as given, and then apply an adjustment that evolves over time in a stochastic way. (For further, brief details, see Appendix B.) Lee and Yang's approach has been continued in later work by Cairns, Blake and Dowd (2006) and Lin and Cox (2005b).

Short-rate models for the development in continuous time of the force of mortality have been proposed by Milevsky and Promislow (2001), Dahl (2004), and Biffis (2005). Milevsky and Promislow (2001) take a more theoretical approach in continuous time which assumes that the force of mortality $\mu(t, x)$ has a Gompertz form $\xi_{0}(t) \exp \left(\xi_{1} x\right)$ where the $\xi_{0}(t)$ term is modelled using a simple mean-reverting diffusion process (see Appendix C). Dahl (2004) works initially in a much more general setting than Milevsky and Promislow. He starts by considering a general diffusion process for the force of mortality before focusing on the parsimonious, affine class of processes and we discuss his approach further in Appendix D. Biffis (2005) extends Dahl's approach to cover jumpdiffusion affine processes.

In contrast to the papers mentioned above, Lin and Cox (2005a) do not propose a specific model for stochastic mortality. Instead, they apply the Wang $(1996,2000,2002,2003)$ transform to convert deterministic projected mortality rates into what might be described as risk-adjusted probabilities. The use of the Wang transform is gaining in popularity in non-life insurance applications where there is a lack of liquidity in the instruments subject to the underlying risks. However, it is not clear from Lin and Cox (2005a) how different

2 The spot mortality rate $q(t, x)$ is the probability at time $t$ that an individual who is aged $x$ and still alive at time $t$ will die before time $t+1$. 
transforms for different cohorts and terms to maturity relate to one another to form a coherent whole.

Another approach to the pricing problem in incomplete financial markets has been proposed by Froot and Stein (1998). They discuss how the capital structure of a financial institution affects the price they are prepared to pay to take on or to offload a non-hedgeable risk. Typically this takes the form of an over-the-counter deal between two institutions. Amongst other results, they demonstrate that the price at which an institution is willing to trade these instruments also depends on how well capitalised it is.

\subsection{Applications of stochastic mortality models}

There are many financial applications in which it is necessary to take account of the stochastic behaviour of mortality. One example is the calculation of quantile (or value-at-risk) reserves for life-office portfolios, where the uncertain future pattern of liability payments will depend, amongst other things, on the future evolution of the force of mortality $\mu(t, x)$. It is also important to take account of stochastic mortality when reserving for policies that incorporate certain types of guarantee.

Taking account of stochastic mortality is also critical when pricing mortality derivatives. Below we provide some examples of mortality derivatives that have been proposed in the literature, not all of which exist in practice at the time of writing:

- Longevity bonds (where coupon payments are linked to the number of survivors in a given cohort). Long-dated longevity bonds (also referred to as survivor bonds: Blake and Burrows, 2001) intended to manage longevity risk have recently been revived by Cox, Fairchild and Pedersen (2000), Blake and Burrows (2001) and Lin and Cox (2005a). Their origin dates back to Tontine bonds issued by a number of European governments in the $17^{\text {th }}$ and $18^{\text {th }}$ centuries. The first modern issue of a longevity bond, with a maturity of 25 years, was announced in November 2004 by the European Investment Bank and BNP Paribas. However, Cox, Fairchild and Pedersen (2000) commented that a number of insurers were proposing to issue longevity bonds as early as 1997. The fact that issues of such bonds were so long in coming suggests that there are substantial practical problems which need to be overcome or that the market is not yet ready to invest in such long-term bonds. (For further details of the EIB/BNP bond, see Cairns, Blake, and Dowd (2005), Cairns, Blake, Dawson and Dowd (2005) and Blake, Cairns and Dowd (2006).)

- Short-dated, mortality-linked securities (market-traded securities whose payments are linked to a mortality index). The first, widely-marketed, bond of this type was issued by Swiss Re at the start of 2004, and it shares characteristics with traditional catastrophe bonds in the non-life insurance markets (see, for example, Schmock, 1999, Lane, 2000, Wang, 2002, and Muermann, 2004). It involves a three-year contract (maturing on 1 January 2007) which 
allows the issuer to reduce its exposure to short-term catastrophic mortality risk. The repayment of the principal is linked to a combined mortality index of experienced mortality rates in five countries (France, Italy, Switzerland, the UK and the USA). Under the contract, the principal will be at risk "if, during any single calendar year in the risk coverage period, the combined mortality index exceeds $130 \%$ of its baseline 2002 level". The credit spread at issue of 135 basis points $(1.35 \%)$ equates to a risk-neutral probability of about 0.04 (that is, $1-(1-0.0135)^{3} \approx 0.04$ ) that the principal would not be repaid at all. This is equivalent to a catastrophic event that would happen, on average, once every 75 years (treating individual years as being independent). The types of catastrophic mortality events that are large enough to breach the threshold include (but are not restricted to) an influenza pandemic, or a natural catastrophe. However, the Swiss Re bond addresses a different type of mortality risk (short-term catastrophic mortality risk) from that considered in this paper (unanticipated long-term changes in population mortality). The catastrophe risks being covered by the Swiss Re bond might be correlated with financial markets (past examples - albeit on a "smaller" scale include 9/11 or the Kobe earthquake in 1995). In contrast, we assume for convenience that systematic mortality risks are independent of the financial markets: an assumption that simplifies the mathematical developments that follow. However, we do discuss briefly how this assumption might be relaxed.

- Survivor swaps (where counterparties swap a fixed series of payments for a series of payments linked to the number of survivors in a given cohort). A small number of survivor swaps have been arranged on an over-the-counter basis. They are not traded contracts and therefore only provide direct benefit to the counterparties in the transaction. The case for survivor swaps is made by Dowd et al (2006).

- Annuity futures (where prices are linked to a specified future market annuity rate). As an example, suppose that $a(t, x)$ represents the market price at time $t$ of a level annuity of $£ 1$ per annum payable monthly in arrears to a male aged $x$ at time $t$. (This might, for example, be a weighted average of the top 5 quotes in the market.) It has been suggested by AFPEN (the association of French Pension Funds) that a traded futures market be set up with $a(t, x)$ (or its reciprocal) as the underlying instrument for selected values of $x$ and with a selection of maturity dates stretching out many years into the future. For a given maturity date, the market could be closed out some months or even a year before the maturity date itself, to reduce the impact, for example, of moral hazard, changes in expensing bases, or the movements of individual annuity providers in and out of the market. For further discussion of annuity futures, see Blake, Cairns and Dowd (2006).

- Mortality options (a range of contracts with option characteristics whose payoff depends on an underlying mortality table at the payment date). For example, a guaranteed annuity option is an investment-linked deferredannuity contract that gives a policyholder the option to convert his accumulated fund at retirement at a guaranteed rate rather than at market annuity 
rates. Market annuity rates are set with reference to both interest rates at retirement and the mortality table in current use by the annuity provider, and this table, of course, depends on mortality forecasts at that time. Contracts of this type are discussed further in Section 4.2. Previous analyses of the role of systematic mortality risk in guaranteed annuity contracts include those of Yang (2001) and Biffis and Millossovich (2004). Milevsky and Promislow (2001) consider both pricing and hedging of contracts involving annuity options. Dahl (2004) applies his model to problems involving insurance contracts with mortality-linked cashflows.

For a more detailed discussion of the types of security that might be issued and the practical issues associated with these contracts, see Blake, Cairns and Dowd (2006).

The more general impact of systematic mortality risk on life insurance has been considered at a theoretical level by Dahl and Møller (2005), while Marocco and Pitacco (1998) and Pitacco (2002) discuss the risk in more general terms. Cowley and Cummins (2005) consider securitisation of life insurance portfolios that incorporate a mixture of risks including mortality.

A final application is the development of optimal asset-allocation strategies in the presence of systematic mortality risk. This type of problem is considered by Dahl and Møller (2005) (who examine optimal hedging) and Yang and Huang (2005) (who examine optimal strategies for defined contribution pension plans).

It is, of course, essential that the evolution of the prices of these derivative contracts should accurately reflect the stochastic evolution of $\mu(t, x)$. The evolution of $\mu(t, x)$ can affect prices in several ways. Most obviously, stochastic mortality has an impact on the value of mortality options: the greater the volatility in mortality rates, the greater is the value of a mortality option (as with financial options). However, the second effect is more subtle and relates to the fact that the 'true' values of financial contracts are often non-linear functions of underlying factors. This point often manifests itself through Jensen's inequality, and an example in the present context would be that the price of a contract based on expected cash flow may not be equal to the value of the contract assuming that mortality follows some central projection. Also (in line with the pricing of financial options) it might manifest itself in our calculating expectations using a different probability measure (denoted below by $Q$ ) from the real-world or true measure (denoted by $P$ ) as, for example, is the case in Dahl (2004). We do not discuss in this paper the many practical issues related to the securitization of mortality risks. These issues are discussed elsewhere (see Cowley and Cummins, 2005, Dowd et al., 2006, Lin and Cox, 2005a, and Blake, Cairns and Dowd, 2006).

\subsection{Choice of reference population}

It is important to note that the reference population underlying the calculation of the mortality rates is critical to both the viability and liquidity of these 
contracts. Some investors (for example, life offices) will wish to use such contracts to help hedge their mortality risk, but if the reference population is inappropriate, they will be exposed to significant basis risk and the mortality derivative might not provide an adequate hedge. Other investors, such as hedge funds, will be attracted to mortality-linked securities because their lack of correlation with other assets helps with the diversification of risk on a general portfolio of investments (see, for example, Cox, Fairchild and Pedersen, 2000). These investors might be less interested in using these derivatives for hedging mortality risk but will be interested in liquidity (liquidity being a key issue as well for speculators). Adequate liquidity will then require a small number of reference populations, but these will need to be chosen carefully to ensure that the level of basis risk is relatively small for those hoping to use the contracts for hedging purposes. For more detail, see Section 2.1.

\subsection{Requirements of a good stochastic mortality model}

Given that mortality is best modelled as a stochastic process, it is reasonable to suppose that any 'plausible' mortality model would meet the following criteria:

- The model should keep the force of mortality positive.

- The model should be consistent with historical data.

- The long-term future dynamics of the model should be biologically reasonable. For example, one might rule out the possibility of an 'inverted' mortality curve (that is, one in which mortality rates for the elderly fall with age). An 'inverted' curve is not only unreasonable a priori, but also conflicts with the normal upward-sloping curves that are always observed in historical data.

- Long-term deviations in mortality improvements from those anticipated should not be mean-reverting to a pre-determined target level, even if this target is time dependent and incorporates mortality improvements. In contrast, short-term deviations from the trend due to local environmental fluctuations might be mean-reverting around the stochastic long-term trend. (We comment further on this below.)

- The model should be comprehensive enough to deal appropriately with the current pricing, valuation or hedging problem.

- It should be possible to value the most common mortality-linked derivatives using analytical methods or using fast numerical methods. However, we stress that this criterion is one of convenience only, and should not be allowed to override the other criteria, which are more important because they are criteria of principle. In other words, we should not drop one of the other criteria merely to obtain an easy (for example, analytical) solution to the problem at hand.

It is worth commenting further on why, specifically, we take the view that long-run stochastic improvements in mortality, $\mu(t, y)$, should not be strongly mean-reverting to some deterministic projection, $\hat{\mu}(t, y)$. The inclusion of mean 
reversion would mean that if mortality improvements have been faster than anticipated in the past then the potential for further mortality improvements will be significantly reduced in the future. In extreme cases, significant past mortality improvements might be reversed if the degree of mean reversion is too strong. Such extreme mean reversion is difficult to justify on the basis of previous observed mortality changes and with reference to our perception of the timing and impact of, for example, future medical advances. Short-term trends might be detected by analysing carefully recent developments in healthcare and in the pharamaceutical industry, but even then the precise, long-term effects of such advances are difficult to judge. As we peer further into the future, it becomes even more difficult to predict what medical advances there might be, when they will happen, and what impacts they will have on survival rates. All of these uncertainties rule out strong mean reversion in a model for stochastic mortality.

Given this list of criteria, it is readily apparent that no one framework dominates the rest: some frameworks fare better by some criteria, and worse by others. There is therefore no strong reason why we should prefer models within any one modelling framework over models in another.

We described above a number of studies that propose specific stochastic models for the future evolution of mortality rates. With the exception of Section 4 , this paper does not offer a new model type. Instead, we seek to set down the general formulation of the problem in continuous time and suggest a range of frameworks for the pricing and valuation of mortality-linked derivatives. Our aim in doing so is to provide, in one place, extensive foundations for the development in the future of further stochastic models for mortality and to ensure that they are used in appropriate ways in pricing problems. To make the exposition easier to follow, we focus our discussion on the problem of pricing new securities. However, we emphasize that the same basic approaches apply to related problems. For example, they apply to the valuation of insurance liabilities that incorporate mortality-linked derivatives, and they also apply (with appropriate changes from risk-neutral to real-world probabilities) to the estimation of risk measures (for example, value at risk) for mortality-related positions.

\subsection{Layout of the paper}

The layout of the paper is as follows. In Section 2, we introduce the fundamental processes for mortality (the force of mortality process $\mu(t, x)$ ) and for the risk-free rate of interest $(r(t))$. These processes feed into survivor indices $S(u, y)$ and a risk-free cash account $C(t)$ that play central roles in our analysis. We work with two fundamental types of financial contract:

- pure endowment contracts (zero-coupon longevity bonds) for a full range of ages and terms to maturity; and

- default-free zero-coupon bonds for a full range of terms to maturity. 
By noting parallels with interest-rate and credit-risk theory, we then describe how pure endowment contracts should be priced if they trade in a perfectly liquid, frictionless and arbitrage-free market ${ }^{3}$.

In this paper we use the terms framework and model in a precise way. Frameworks provide us with general approaches to modelling, and it is frameworks that are the main focus of this paper. We use the word model to mean a particular formulation developed within a given framework. For example, we have the Vasicek (1977), Cox, Ingersoll and Ross (1985), and Black and Karasinski (1991) models within the short-rate modelling framework. The framework normally defines what we can use as the basic building blocks in the modelling process.

In Section 3 we go on to describe briefly different frameworks that could be employed to build up models for stochastic mortality with further detail given in the Appendices. Subsection 3.1 pulls together all of the short-rate models described above under the one short-rate-modelling framework and discusses how these models can be used to build an arbitrage-free market in mortality derivatives. Subsections 3.2 to 3.4 then discuss various other modelling frameworks that have received much less attention. From these, the most novel contribution in this paper is contained in the separate Section 4 where we propose a number of different market models for mortality. Section 5 concludes.

Each of these frameworks is drawn from the field of interest-rate modelling but with the risk-free rate of interest replaced by the force of mortality. These are all described in theoretical terms: and, with the exception of Section 4, no specific models are proposed or analysed. Rather, the aim is to give readers a choice of frameworks within which they can build their own continuous-time stochastic mortality models. Most models built up within one framework can be reformulated within any of the other frameworks (in the same way, for example, that the Vasicek, 1977, short-rate model for $r(t)$ can be re-expressed as a forward-rate model). This said, it is also the case that most models rest naturally within one particular framework, and are awkward to express within the others.

\section{THE TERM STRUCTURE OF MORTALITY}

In this section, we will define the basic components of a model for stochastic mortality. We start by considering the force of mortality, $\mu(t, x)$, at time $t$ for individuals aged $x$ at time $t$. Traditional static mortality models implicitly assume that $\mu(t, x) \equiv \mu(x)$ for all $t$ and $x$, while deterministic mortality projections imply that $\mu(t, x)$ is a deterministic function of $t$ and $x$. By contrast, the models we will consider here will treat $\mu(t, x)$ as a stochastic process.

\footnotetext{
3 Naturally, we do not claim that real-world markets are perfectly liquid or frictionless. However, we can state that if prices are calculated in the way proposed then even an illiquid market with frictions will be arbitrage-free. Conversely, if we were to propose a pricing framework that violates the conditions in Section 2, then the possibility of arbitrage would emerge over time as the market becomes more liquid or trading costs begin to fall.
} 
There are two types of stochastic mortality (see, for example, Dahl, 2004):

- The first is specific (or unsystematic) mortality risk - the risk that the actual number of deaths deviates from the anticipated number because of the finite number of lives in a given cohort. This type of risk can largely be diversified by investors under the usual assumption that future lifetimes for different individuals are independent random variables. Specific mortality risk does not lead to a risk premium in the price of mortality derivatives.

- Systematic mortality risk - the risk that the force of mortality evolves in a different way from that anticipated. This type of risk cannot be diversified away and therefore leads to the incorporation of a risk premium.

By drawing parallels with the pricing of financial contracts, we might expect that, with mortality derivatives, systematic mortality risk should be priced using a risk-neutral probability measure, $Q$, which is different from the realworld probability measure, $P$ (sometimes also called the objective or physical measure). This intuition turns out to be correct: we will argue below that mortality derivatives need to be priced with reference to such a measure in order for the market to be arbitrage free. We stress at this point, though, that the risk-neutral measure $Q$ might not be uniquely determined due to market incompleteness. Instead the choice of $Q$ becomes part of the modelling process. An important additional observation is that the explicit use of a risk-neutral measure, $Q$, for pricing - even if the market is highly illiquid or has high transaction costs - ensures that the market will be free from riskless arbitrage (Fundamental Theorem of Asset Pricing). As mortality-linked securities begin to emerge and as we gather market price data we can then test for the validity of our assumptions about $Q$. For further discussion of the relationship between $P$ and $Q$, the reader is referred to Dahl (2004), Cairns, Blake and Dowd (2006) and Biffis (2005).

One example where prices might be calculated with reference to a risk-neutral measure is the Swiss Re mortality bond. The 135 basis point spread equates to a risk-neutral probability, approximately, of 0.0135 per annum of default: that is, that the principal will not be paid out. However, the real-world probability is thought to be rather less than this (see, for example, Beelders and Colarossi, 2004).

\subsection{Basic building blocks}

We have previously indicated that our aim is to develop a set of theoretical frameworks to price mortality derivatives. In order to do so, we will make the convenient but over-simplifying assumption that the force of mortality at time $t, \mu(t, y)$, is observable at time $t$ for all $y$. In reality, we can only estimate $\mu(t, y)$ from a finite amount of data, and this estimate is only calculated and published some months or years after the event. The length of this delay also depends considerably on the reference population: for example, the UK industry-wide 
Continuous Mortality Investigation tables take longer to compile than tables relating to one specific life office. We recognise that these are important practical issues but we will leave them for future work.

\subsubsection{The survivor index}

We will use as our basic building block a family of index-linked zero-coupon longevity bonds. The indices we will employ are related to survival probabilities for different ages. Thus we define the survivor index

$$
S(u, y)=\exp \left(-\int_{0}^{u} \mu(t, y+t) d t\right) .
$$

If $\mu(t, x)$ is deterministic then $S(u, y)$ is equal to the probability that an individual aged $y$ at time 0 will survive to age $y+u$. Similarly, the probability that an individual aged $x$ at time $t_{1}$ will survive until a later time $t_{2}$ is $S\left(t_{2}, x-t_{1}\right) / S\left(t_{1}, x-t_{1}\right)$.

In this paper, we are mainly concerned with models in which $\mu(t, x)$ is stochastic. Looking forward from time 0 , this means that $S(u, y)$ is now a random variable. In this case, $S(u, y)$ can still be regarded as a survival probability, albeit one that can only be observed at time $u$ rather than at time 0 . However, it is straightforward to extract a survival probability by taking the expectation of the random variable $S(t, x)$ (equation (2.2) below). We prove this by using a combination of indicator random variables and conditional expectation. Thus, consider an individual aged $x$ at time 0 . Let $Y_{x}(u)$ be a Markov chain which is equal to 1 if the individual is still alive at time $u$. Also let $\mathcal{M}_{t}$ be the filtration (or information) generated by the evolution of the term-structure of mortality, $\mu(u, x)$, up to time $t$ (that is, $\mathcal{M}_{t}$ gives us full information about changes in mortality up to and including time $t$, but no information about how mortality rates will develop after time $t$ ). The real-world or true survival probability measured at time 0 , that an individual aged $x$ at time 0 survives until time $u$ is

$$
p_{P}(0, u, x)=E_{P}\left[Y_{x}(u)\right]=E_{P}\left[E_{P}\left\{Y_{x}(u) \mid \mathcal{M}_{u}\right\}\right]=E_{P}[S(u, x)] .
$$

Remark 2.1.1. Note that $E_{P}[S(u, x)]>\exp \left[-\int_{0}^{u} E_{P}(\mu(t, y+t)) d t\right]$ by Jensen's inequality. Also, if $\bar{\mu}(t, y+t)$ is a deterministic best estimate at time 0 of future mortality (for example, the median) then we will still usually have $E_{P}[S(u, x)] \neq$ $\exp \left[-\int_{0}^{u} \bar{\mu}(t, y+t) d t\right]$.

More generally, we can define the survival probabilities at time $t$ as follows. Let $p_{P}(t, u, x)$ be the probability under $P$ that an individual aged $x$ at time 0 and still alive at the current time $t$ survives until time $u$ :

$$
p_{P}(t, u, x)=E_{P}\left[Y_{x}(u) \mid Y_{x}(t)=1, \mathcal{M}_{t}\right]=E_{P}\left[\frac{S(u, x)}{S(t, x)} \mid \mathcal{M}_{t}\right] .
$$


For the alternative risk-neutral probability measure $Q$, we can define the corresponding survival probabilities:

$$
p_{Q}(t, u, x)=E_{Q}\left[Y_{x}(u) \mid Y_{x}(t)=1, \mathcal{M}_{t}\right]=E_{Q}\left[\frac{S(u, x)}{S(t, x)} \mid \mathcal{M}_{t}\right] .
$$

Remark 2.1.2. In deriving this last expression, we have relied on the conventional assumption that non-systematic mortality risk does not attract a risk premium. Even when $S(u, x) / S(t, x)$ is known, there is a range of equivalent probability measures for the Bernoulli random variable $\left(Y_{x}(u) \mid Y_{x}(t)=1, \mathcal{M}_{u}\right)$. However, only one of these is consistent with the absence of a risk premium for the non-systematic mortality risk: specifically $\operatorname{Pr}_{Q}\left[Y_{x}(u)=1 \mid Y_{x}(t)=1, \mathcal{M}_{u}\right]=S(u, x) / S(t, x)$.

\subsubsection{Zero-coupon longevity bonds}

We are now in a position to consider the pricing of index-linked zero-coupon longevity bonds. There is (potentially) a different bond for each maturity date $T$ and for each age $x$ at time 0 . We refer to a specific bond as the $(T, x)$-bond for compactness.

The $(T, x)$-bond pays the amount $S(T, x)$ at time $T$. This payment is well defined in the sense that $S(T, x)$ is an observable quantity at time $T$. The $(T, x)$ bond is an example of what financial mathematicians call a tradeable asset (also known as a pure-discount asset to some financial economists): that is, an asset that pays no coupons or dividends and whose price, relative to the issue price, at any time $t<T$ represents the total return on an investment in that asset. For an asset that does pay dividends or coupons a tradeable asset can be created by reinvesting the dividends in the underlying asset itself.

To price such bonds, we need to use the term-structure of interest rates. Let $P(t, T)$ represent the price at time $t$ of a zero-coupon bond that pays 1 at time $T$. The instantaneous forward rate curve at time $t$ is given by

$$
f(t, T)=-\frac{\partial}{\partial T} \log P(t, T)
$$

and the instantaneous risk-free rate of interest is

$$
r(t)=\lim _{T \rightarrow t} f(t, T)
$$

(see, for example, Cairns, 2004b).

A cash (or money-market) account accrues at the risk-free rate of interest. Its value at time $t$ is denoted by $C(t)$ with

$$
\begin{aligned}
d C(t) & =r(t) C(t) d t \\
\Rightarrow C(t) & =C(0) \exp \left(\int_{0}^{t} r(u) d u\right) .
\end{aligned}
$$


Let $\mathcal{F}_{t}$ be the filtration generated by the term-structure of interest rates up to time $t$, and $\mathcal{H}_{t}$ be the combined filtration for both the term-structure of interest rates and mortality.

Now let $\tilde{B}(t, T, x)$ represent the price at time $t$ of the $(T, x)$-bond that pays $S(T, x)$ at time $T$. If there exists a measure $Q$ (the risk-neutral measure) equivalent to the real-world measure $P$ with

$$
\begin{aligned}
P(t, T) & =E_{Q}\left[\frac{C(t)}{C(T)} \mid \mathcal{F}_{t}\right] \\
\text { and } \tilde{B}(t, T, x) & =E_{Q}\left[\frac{C(t)}{C(T)} S(T, x) \mid \mathcal{H}_{t}\right]
\end{aligned}
$$

for all $t, T$ and $x$ (which implies that $P(t, T) / C(t)$ and $\tilde{B}(t, T, x) / C(t)$ are $Q$-martingales) then the dynamics of the combined financial market are arbitrage free. Equation 2.5 matches those of Milevsky and Promislow (2001) and Dahl (2004) and encompasses the full range of models including those with more than one source of randomness.

\section{Assumption 1}

We now make the convenient assumption that, under the risk-neutral measure $Q$, the dynamics of the term structure of mortality are independent of the dynamics of the term-structure of interest rates. This assumption simplifies the discussion and allows us to present the modelling analogies with the maximum possible clarity. However, it is not essential that we make this assumption and it can be relaxed.

Whether interest rates and mortality rates are related is a topic for debate: many economists are sceptical, but others point to anecdotal evidence that suggests some form of relationship. Should one believe that interest and mortality rates are dependent, one might model it along lines such as those pursued by Miltersen and Persson (2005), who present a forward-mortality framework that explicitly allows for interest and mortality to be related.

A key consequence of this independence assumption is that it allows us to separate the pricing of mortality risk from the pricing of interest-rate risk. It follows that

$$
\begin{aligned}
& \tilde{B}(t, T, x)=E_{Q}\left[\frac{C(t)}{C(T)} \mid \mathcal{F}_{t}\right] E_{Q}\left[S(T, x) \mid \mathcal{M}_{t}\right] \\
& =P(t, T) B(t, T, x) \\
& \text { where } B(t, T, x)=E_{Q}\left[S(T, x) \mid \mathcal{M}_{t}\right] \text {. }
\end{aligned}
$$

Thus $B(t, T, x)$ is a martingale under $Q$. We can also assume that the $B(t, T, x)$ processes are strictly positive (which is equivalent to ruling out the possibility of catastrophic events that wipe out the entire population). This allows us to make three further observations: 
- $B(t, T, x) / B(t, t, x)=p_{Q}(t, T, x)$. Since we can regard the $B(t, T, x)$ as spot estimates, we will refer to the $p_{Q}(t, T, x)$ as spot survival probabilities.

- We can use the $B(t, T, x)$ to define the forward force of mortality surface (see, for example, Milevsky and Promislow, 2001, and Dahl, 2004) (we will sometimes shorten this to forward mortality surface):

$$
\bar{\mu}(t, T, x+T)=-\frac{\partial}{\partial T} \log B(t, T, x) .
$$

Conversely, knowledge of the forward mortality surface allows us to price the bonds:

$$
\frac{B(t, T, x)}{B(t, t, x)}=\exp \left[-\int_{t}^{T} \bar{\mu}(t, u, x+u) d u\right] .
$$

If we take $T=t$, we get the spot force of mortality:

$$
\mu(t, x+t)=\bar{\mu}(t, t, x+t) .
$$

(A more general form for the forward force of mortality surface has been defined in the parallel work of Miltersen and Persson (2005). In their formulation, interest and mortality rates are allowed to be dependent, so that separation of $\tilde{B}(t, T, x)$ into $P(t, T) \times B(t, T, x)$ is not possible without resorting to the use of forward measures equivalent to $Q$. They define $\bar{\mu}(t, T, x+T)=$ $-\frac{\partial}{\partial T} \log \tilde{B}(t, T, x)-f(t, T)$, where $f(t, T)$ is the forward interest rate $-\frac{\partial}{\partial T} \log$ $P(t, T)$. If Assumption 1 holds then Miltersen and Persson's definition is equivalent to that in equation (2.6).)

It is also convenient at this point to define the forward survival probability: $p_{Q}\left(t, T_{0}, T_{1}, x\right)=$ the probability, based on the information about mortality rates available at time $t, \mathcal{M}_{t}$, that an individual alive and aged $x+T_{0}$ at time $T_{0}$ will survive until time $T_{1}$. This definition implies that

$$
p_{Q}\left(t, T_{0}, T_{1}, x\right)=\exp \left[-\int_{T_{0}}^{T_{1}} \bar{\mu}(t, u, x+u) d u\right]=\frac{B\left(t, T_{1}, x\right)}{B\left(t, T_{0}, x\right)} .
$$

- Let us assume that the dynamics of the term structure of mortality are governed by an $n$-dimensional Brownian motion $\tilde{W}(t)$ under $Q$. The martingale property of $B(t, T, x)$ together with its positivity now allows us to write down the stochastic differential equation for $B(t, T, x)$ in the following form

$$
d B(t, T, x)=B(t, T, x) V(t, T, x)^{\prime} d \tilde{W}(t)
$$

where $V(t, T, x)$ is a family of previsible ${ }^{4}$ vector processes that specify the volatility term structure of the $B(t, T, x)$.

4 To describe a process, $X(t)$, as previsible means that the value of $X(t)$ is known or observable by time $t$. 
We will now consider the possible frameworks which we can use to model the dynamics of the $B(t, T, x)$ processes. These correspond to a variety of frameworks used in modelling interest rates (see, for example, Cairns, 2004b):

- short-rate modelling framework for the dynamics of $\mu(t, y)$ (which correspond to short-rate models for the risk-free rate of interest, $r(t)$, including those of Vasicek, 1977, Cox, Ingersoll and Ross, 1985, and Black and Karasinski, 1991);

- forward-mortality modelling framework for the dynamics of the forward mortality surface, $\bar{\mu}(t, T, x+T)$ (corresponding to the framework of Heath, Jarrow and Morton, 1992);

- positive-mortality modelling framework for the spot survival probabilities, $p_{Q}(t, T, x)$ (corresponding to the positive-interest framework developed by Flesaker and Hughston, 1996, Rogers, 1997, and Rutkowski, 1997);

- market modelling framework for forward survival probabilities or forward annuity prices (corresponding to the LIBOR and swap market models of Brace, Gatarek and Musiela, 1997, and Jamshidian, 1997).

We also include a short section that discusses the parallels between pricing mortality and credit derivatives. In doing so, we also review the similarities that allow us to apply some of the intensity-based models that have been developed in the credit-pricing literature to mortality problems.

We stress that the purpose of the following sections is to pull together the theoretical foundations of this relatively new field. We leave for further work the development of specific new models within the different frameworks considered here. We also leave for others the process of fitting these models to historical and market data: such a task requires the use of estimation techniques that are tailored to individual models and goes well beyond the scope of the present paper.

\section{REVIEW OF EXISTING MODELLING FRAMEWORKS}

\subsection{Short-rate modelling framework}

Models built up within this framework specify directly the dynamics of $\mu(t, y)$. Existing models for the term-structure of mortality within this framework include Lee and Carter (1992), Lee (2000a), Yang (2001), Brouhns, Denuit and Vermunt (2002), Renshaw and Haberman (2003), and Cairns, Blake and Dowd (2006) in discrete time, and Milevsky and Promislow (2001) and Luciano and Vigna (2005) in continuous time. Milevsky and Promislow (2001) exploit the completeness of the market in their model to calculate both prices and hedging strategies for an annuity option. Dahl (2004) develops the general one-factor version of equation (3.1) below and then focuses on the affine class of processes as one that gives an analytical solution for $p_{Q}(t, T, x)$. In continuous time, we 
model the force of mortality which will have the general stochastic differential equation

$$
d \mu(t, y)=a(t, y) d t+b(t, y)^{\prime} d \tilde{W}(t)
$$

where $a(t, y)$ and $b(t, y)$ (an $n \times 1$ vector) are previsible processes and $\tilde{W}(t)$ is a standard $n$-dimensional Brownian motion under the risk-neutral measure $Q^{5}$. We then have (see, for example, Milevsky and Promislow, 2001, or Dahl, 2004) ${ }^{6}$

$$
\begin{aligned}
\frac{B(t, T, x)}{B(t, t, x)} & =p_{Q}(t, T, x) \\
& =E_{Q}\left[\exp \left(-\int_{t}^{T} \mu(u, x+u) d u\right) \mid \mathcal{M}_{t}\right] .
\end{aligned}
$$

We can make the following observations about this framework:

- We have specified that $\tilde{W}(t)$ and $b(t, y)$ are $n \times 1$ vectors. This means that we can allow for the possibility that short-term changes in the term-structure of mortality can be different at different ages: that is, mortality changes at different ages are not perfectly correlated. Different rates of change at different ages can also be achieved through the $a(t, y)$ drift function.

- $a(t, y)$ and $b(t, y)$ might depend on other diffusion processes which are themselves adapted to $\mathcal{M}_{t}$. Note that this dependence allows $b(t, y) \equiv 0$, in which case the force of mortality curve evolves in a smooth fashion over time. However, the evolution of the force of mortality curve is still stochastic because of its dependence on the stochastic drift rate $a(t, y)$. Other models might assume that $b(t, y) \neq 0$, in which case the force of mortality curve exhibits a degree of local volatility.

- The assumption that $b(t, y) \equiv 0$ is equivalent to the assumption that the volatility function $V(t, T, x)$ for the $B(t, T, x)$ processes, and their first derivatives with respect to $T$, tend to zero as $T \rightarrow t$. Thus, the shortest-dated bonds will have a very low volatility.

- This framework also includes models that assume that $\mu(t, y)$ takes some parametric form (for example, the Gompertz-Makeham model $\mu(t, x)=\xi_{0}(t)$ $\left.+\xi_{1}(t) e^{\xi_{2}(t) x}\right)$. We can model the parameters in this curve as diffusion processes. This class is a specific example of the type noted above where $a(t, y)$ and $b(t, y)$ themselves depend on other diffusion processes.

5 The dynamics expressed in (3.1) have been extended by Biffis (2005) to include jumps as well as a diffusion.

6 If we are modelling the spot survival probabilities, $p_{Q}(t, t+1, x)$, or the spot mortality rates, $q_{Q}(t, t+1$, $x)=1-p_{Q}(t, t+1, x)$ in discrete time, then the equivalent of equation (3.2) in discrete time is $p_{Q}(t, T, x)=$ $E_{Q}\left[p_{Q}(t, t+1, x) \times \ldots \times p_{Q}(T-1, T, x) \mid \mathscr{M}_{t}\right]$. 
The framework includes the affine class of models for $\mu(t, x)$ considered by Dahl (2004) (with mean reversion around a deterministic projection) under which the spot survival probabilities have the closed form

$$
p_{Q}(t, T, x)=\exp \left[A_{0}(t, T, x)-A_{1}(t, T, x) \mu(t, x+t)\right]
$$

with $n=1$ dimension. Dahl provides sufficient conditions on $a(t, y)$ and $b(t, y)$ (equation (3.1)) that result in this affine representation for $p_{Q}(t, T, x)$. These conditions mimic those of Duffie and Kan (1996) for interest-rate models.

An approach that generalises equation (3.1) to what is more truly a bidimensional process has been proposed by Biffis and Millosovich (2005). Their model for $\mu(t, y)$ is driven by a Markov random field (within which our finitedimensional Brownian motion is a special case). A specific example for $\mu(t, y)$ suggested by Biffis and Millossovich is linked to a Markov-random-field version of the autoregressive quadratic term structure model investigated by Ahn, Dittmar and Gallant (2002) and Rogers (1997) (see also Cairns, 2004b).

\subsection{Forward mortality modelling framework}

The next set of models are forward mortality models.

\subsubsection{Risk-neutral dynamics}

Suppose that we have the two stochastic differential equations:

$$
\begin{aligned}
d B(t, T, x) & =B(t, T, x) V(t, T, x)^{\prime} d \tilde{W}(t) \\
\text { and } d \bar{\mu}(t, T, x+T) & =\alpha(t, T, x+T) d t+\beta(t, T, x+T)^{\prime} d \tilde{W}(t)
\end{aligned}
$$

where $V(t, T, x), \alpha(t, T, x+T)$ and $\beta(t, T, x+T)$ are previsible processes. Now we might ask if we can specify $V(t, T, x), \alpha(t, T, x+T)$ and $\beta(t, T, x+T)$ freely. However, by drawing parallels with the forward-interest-modelling framework of Heath, Jarrow and Morton (1992) (HJM), we can see that there will need to be some form of relationship between $V(t, T, x), \alpha(t, T, x+T)$ and $\beta(t, T, x+T)$ to be sure that we have an arbitrage-free framework for pricing mortality-linked derivatives. Before we develop the mathematical form of this relationship we can remark that the presence of age as an additional dimension means that our framework provides a richer and more complex modelling environment than does the classical HJM framework.

One can use a similar argument to Heath, Jarrow and Morton (1992) to show that for the model dynamics to be arbitrage free we require

$$
\alpha(t, T, x+T)=-V(t, T, x)^{\prime} \beta(t, T, x+T) .
$$


The mathematical argument that leads to this identity focuses on a single age $x$ at time 0 . However, since the mortality dynamics of all cohorts are driven by the same $n$-dimensional Brownian motion $d \tilde{W}(t)$, we can see that (3.5) must apply to all ages $x$, otherwise there would be arbitrage between cohorts.

As already noted, a more general approach to modelling of the forward mortality surface has been taken by Miltersen and Persson (2005) which includes dependence between interest and mortality rates. This results in a more complex expression for the drift $\alpha(t, T, x+T)$ than that in (3.5).

As with the other frameworks, the challenge is to specify an appropriate form for $\beta(t, T, x+T)$ or $V(t, T, x)$. The chosen formulation needs to ensure that the forward mortality surface remains strictly positive. This requirement is most easily achieved by making $\beta(t, T, x+T)$ explicitly dependent on the current forward mortality surface. In addition, the chosen form needs to ensure that the spot force of mortality curve, $\mu(t, y)$, retains an appropriate shape (for example, that it is increasing with age).

An alternative approach to modelling forward mortality rates has been developed by Olivier and Smith (see Olivier and Jeffery, 2004, and Smith, 2005). They work with the one-year forward survival probabilities $p_{Q}(t, T-1, T, x)$ and exploit the martingale property of $E_{Q}\left[S(T, x) \mid \mathcal{M}_{t}\right]$ and the analytical properties of the Gamma distribution to derive arbitrage-free dynamics.

\subsubsection{Real-world dynamics}

In Sections 3.1 and 3.2.1, we considered dynamics under the risk-neutral measure. This is appropriate for pricing but it is not appropriate for risk measurement purposes where we require true or real-world probabilities. This can be achieved by incorporating a market price of risk $\gamma_{W}(t)$, a previsible process. In the forward modelling context this means we replace $d \tilde{W}(t)$ in equations (3.3) and (3.4) by $d W(t)+\gamma_{W}(t) d t$ to get, for example, $d B(t, T, x)=B(t, T, x) V(t, T, x)^{\prime}(d W(t)+$ $\gamma_{W}(t) d t$ ). Besides allowing us to measure risk using real-world probabilities, it also allows us to separate the risk premium on a mortality-linked security into risk premiums linked to interest-rate risk and mortality risk. Where there is dependence between interest and mortality rates (Miltersen and Persson, 2005) this risk premium might even be decomposed into three parts: the pure interestrate risk premium as a result of the discount factor, a risk premium resulting from correlation between the underlying and interest rates, and a risk premium resulting from independent (specific) mortality risk.

\subsection{The positive-mortality framework}

We now turn to our third class of models, the positive mortality framework (see, for example, Rogers, 1997, and Rutkowski, 1997).

Let $\tilde{P}$ be some measure equivalent to $Q$, and let $A(t, x)$ be some family of $\mathcal{M}_{t}$ adapted, strictly-positive supermartingales. 
Define

$$
p_{Q}(t, T, x)=\frac{B(t, T, x)}{B(t, t, x)}=\frac{E_{\vec{P}}\left[A(T, x) \mid \mathcal{M}_{t}\right]}{A(t, x)} .
$$

The strict positivity of $A(t, x)$ means that $p_{Q}(t, T, x)$ is positive. The supermartingale property of $A(t, x)$ ensures that the $p_{Q}(t, T, x)$ are less than or equal to 1 and decreasing in $T>t$ (so the force of mortality is always positive). It is straightforward to demonstrate (for example, through the application of the Radon-Nikodym derivative $d Q / d \tilde{P})$ that the resulting dynamics of $B(t, T, x)$ are appropriate for an arbitrage-free pricing model (see, also, Rogers, 1997, and Rutkowski, 1997). Within this pricing framework, the drift of $A(t, x)$ under $\tilde{P}$ is equal to $-\mu(t, x+t) \times A(t, x)$. (In the corresponding positive-interest model the drift of $A(t)$ is equal to $-r(t) \times A(t)-$ see, for example, Cairns, 2004b.)

Equation (3.6) appears deceptively simple as a pricing formula. However, the effort comes in specifying a model for the processes $A(t, x)$ and in calculating the expectations. In particular, besides the requirement that, for each $x$, $A(t, x)$ be a supermartingale, all of the $A(t, x)$ must evolve in a way that results in a biologically reasonable form for $\mu(t, x+t)$ at each $t$. (For examples in interest-rate modelling, see Flesaker and Hughston, 1996, Rogers, 1997, Cairns, 2004a, and Cairns and Garcia Rosas, 2004.)

A special case of this framework is an adaptation of Flesaker and Hughston (1996) (FH). Let $N(t, s, x)$, for $0<t<s$, be a family of strictly-positive martingales under $\tilde{P}$. Define

$$
A(t, x)=\int_{t}^{\infty} N(t, s, x) d s
$$

The martingale property of $N(t, s, x)$ means that

$$
\begin{aligned}
E_{\tilde{P}}\left[A(T, x) \mid \mathcal{M}_{t}\right] & =\int_{T}^{\infty} N(t, s, x) d s \\
& <A(t, x) .
\end{aligned}
$$

It follows from (3.8) that $A(t, x)$ satisfies the Rogers/Rutkowski requirements for a strictly-positive supermartingale.

Combining equations (3.7) and (3.6) we now get

$$
p_{Q}(t, T, x)=\frac{\int_{T}^{\infty} N(t, s, x) d s}{\int_{t}^{\infty} N(t, s, x) d s} .
$$

From a computational point of view this involves, at worst, the numerical evaluation of a one-dimensional integral, no matter what the model for $N(t, s, x)$ 
or the number of factors involved. Our problem is now one of devising an appropriate model for the family of martingales $N(t, s, x)$.

It is common in interest-rate-derivatives markets to calibrate the initial term structure of the model to the observed interest-rate term structure, and we can apply this approach to the mortality term structure too. Suppose then that we take as given at time 0 the market prices of the zero-coupon bonds, $P(0, T)$, and the $(T, x)$-bonds, $\tilde{B}(0, T, x)$, for all $x$ and $T>0$. From this, we can derive the implied spot survival probabilities $p_{Q}(0, T, x)=\tilde{B}(0, T, x) / P(0, T)$. The initial values for the family $N(t, T, s)$ can then be calibrated as follows:

$$
N(0, T, x)=-\frac{\partial}{\partial T} p_{Q}(0, T, x)=\bar{\mu}(0, T, x+T) p_{Q}(0, T, x) .
$$

This initial calibration is unique up to a strictly-positive, constant scaling factor.

By analogy with interest-rate modelling, this framework might contain natural model formulations that are difficult to identify in other frameworks. For example, the Cairns (2004a) interest-rate model can be reformulated as a short-rate model. However, the short-rate formulation is rather clumsy compared with the positive-interest formulation.

\subsection{Credit-risk modelling framework}

In this review section we consider, finally, credit-risk models.

Artzner and Delbaen (1995), Milevsky and Promislow (2001) and Biffis (2005) noted that the zero-coupon longevity bond with price $\tilde{B}(t, T, x)$ at time $t$ is similar to a zero-coupon corporate bond that pays 1 at $T$ if there has been no default and 0 if the bond has defaulted. There are many models that address the problem of how to price such bonds (see, for example, the textbooks by Schönbucher, 2003, or Lando, 2004). In the present context, the most useful models for default risk that could be translated into a stochastic mortality model are intensity-based models (see, for example, Schönbucher, 2003, Chapter 7). In these models the default intensity, $\lambda(t)$ corresponds to the force of mortality $\mu(t, x+t)$. This similarity between the force of mortality and the default intensity allows us to apply intensity-based models from the credit-risk literature to the pricing of mortality instruments.

However, there are some differences between mortality risk and credit risk that will be reflected in the type of model used:

- In a credit risk context different companies are equivalent to different cohorts in the mortality model. In mortality modelling there is a natural ordering of the different cohorts (that is, by current age) with strong correlations between adjacent cohorts. In contrast, there is no natural ordering of individual companies and consequently, although defaults may be correlated, there is no natural counterpart to the additional age-related structure in a mortality model. 
- The default intensity in a credit model is likely to be modelled as a meanreverting process that is also possibly time-homogeneous. In contrast, mortality models are certainly time inhomogeneous and need to incorporate non-mean-reverting elements. This has the important implication that Cox, Ingersoll and Ross (1985)-type models can be used for credit-risk models, but not for mortality-based models.

- The default intensity is likely to be correlated with the interest-rate term structure, whereas the force of mortality is unlikely to be.

We can conclude that credit-risk modelling does have something to offer us in the mortality context. However, we need to use models that reflect the differences described above, or else we must adapt suitable credit-risk models to handle mortality risk.

Remark 3.4.1. In Remark 2.1.2, we noted that once the development of $\mu(t, x+t)$ is known, the date of death of an individual does not attract a further risk premium since this risk is diversifiable. In considering credit risk, we cannot assume that default timings are independent and that this risk (once the default intensities have been established) is diversifiable. Credit risk is, therefore, more general than stochastic mortality modelling in that the modelling of the default event can be subject to a risk premium, even when the default intensity is known.

\section{MORTALITY MARKET MODELLING FRAMEWORK}

We turn now to the mortality market models. This section describes the first development of a stochastic mortality market model in the same spirit as the market models used in interest-rate modelling. We begin with some preliminaries about the types of model covered by this framework.

As with the previous frameworks, market models are formulated in continuous time. However, in contrast to the previous frameworks, market models give us the dynamics for a restricted set of assets or forward rates (for example, the prices $\tilde{B}(t, T, x)$ for $T \in\{1,2,3, \ldots\})$. The prices of other assets not modelled might be inferred by interpolation. However, these inferences are not exact, since the market is incomplete outside of the market variables that are modelled explicitly.

Within an interest-rate context, one of the key steps in the development of a market model (see, for example, Miltersen, Sandmann and Sondermann, 1997, Brace, Gatarek and Musiela, 1997, and Jamshidian, 1997) is making a change from the traditional risk-neutral probability measure $Q$ to a different and moresuitable pricing measure. This is done by changing the numeraire from cash to a different tradeable asset. For example, with the LIBOR market model, we use a zerocoupon bond price, $P(t, T)$, as the numeraire. In this section we will discuss first a possible change of numeraire in the mortality-modelling context and then show how this change of numeraire can be used to develop mortality market models. 


\subsection{Change of numeraire}

To begin our discussion, we simplify matters by placing ourselves in a zerointerest environment. In this case, the $B(t, T, x)$ represent the price processes for a set of tradeable assets, since $B(t, T, x)=\tilde{B}(t, T, x)$. Recall that the processes $B(t, T, x)$ are martingales under $Q$ with SDE's

$$
d B(t, T, x)=B(t, T, x) V(t, T, x)^{\prime} d \tilde{W}(t)
$$

for appropriate previsible volatility functions $V(t, T, x)^{7}$.

Now consider some, strictly-positive, tradeable assets as numeraires. As a specific first example, consider $B(t, \tau, y)$ as the numeraire. We then consider processes of the type

$$
Z(t, T, x)=\frac{B(t, T, x)}{B(t, \tau, y)}
$$

For most problems, it is likely that the most productive choice of $y$ will be $x$ itself (since then $Z(\tau, \tau, x)=p_{Q}(\tau, T, x)$ ). If we then apply Ito's formula and the product rule, we find that

$$
d Z(t, T, x)=Z(t, T, x)(V(t, T, x)-V(t, \tau, x))^{\prime}(d \tilde{W}(t)-V(t, \tau, x) d t) .
$$

Now define a new process $W^{\tau, x}(t)=\tilde{W}(t)-\int_{0}^{t} V(s, \tau, x) d s$. Provided that $V(t, \tau, x)$ satisfies the Novikov condition, we can use the Girsanov theorem (see, for example, Karatzas and Shreve, 1998) to infer that there exists a measure $P_{\tau, x}$ equivalent to $Q$ under which $W^{\tau, x}(t)$ is a standard Brownian motion. In this case

$$
d Z(t, T, x)=Z(t, T, x)(V(t, T, x)-V(t, \tau, x))^{\prime} d W^{\tau, x}(t),
$$

so that $Z(t, T, x)$ is a martingale under $P_{\tau, x}$.

In what follows, we will make more sophisticated choices for the numeraire, but the basic techniques described above will remain the same.

\subsection{The annuity market model}

In this section we will consider how to model the price processes underpinning a specific type of insurance contract, the deferred annuity. In its simplest form a deferred annuity contract promises to pay a fixed annuity for life from some future date in return for the payment of a single premium at the commencement of the policy.

7 Readers who are familiar with interest-rate market models can consider the cash (or money-market) account (equation 2.3) as being the numeraire when pricing under $Q$. In this zero-interest-rate environment, the cash account is equal to 1 for all time. 
Variations on this basic contract are better described as savings contracts that are used at a future date to purchase an annuity at the prevailing rates at that time. The simplest form of this contract promises to deliver a lump sum of, say, $£ 1000$ at time $T$ which is then converted at market rates into an annuity. In the UK and many other countries, contracts of this type were often accompanied in the late 20th century by a guarantee that the resulting annuity would be no less than some guaranteed minimum. We will consider in this section how to value this contract. The approach that we take here is also in the spirit of the Pelsser (2003) analysis of the pricing of guaranteed annuity options. However, Pelsser concentrates on the inherent interest-rate and equity risks, whereas we concentrate here on mortality and interest-rate risks.

To begin, we consider the following linear (that is, option-free) forward annuity contract:

- The contract is issued at time $t$ to $N(t)$ identical individuals (the policyholders) who are all age $x+t$ at time $t$.

- No money is exchanged at time $t$.

- At time $T>t$, there are $N(T)$ survivors out of the original $N(t)$.

- Each surviving policyholder at $T$ must pay $£ 1$ and in return each will receive a level annuity of $£ F$ per annum payable at times $T+1, T+2, \ldots$ for as long as the policyholder is still alive (that is payments cease immediately on death).

- If a policyholder dies before time $T$, then the contract immediately expires worthless.

When $t=T$ the forward annuity rate $F(T, T, x)$ is equal to the immediate annuity rate: that is, each $£ 1$ of premium at $T$ buys an annuity of $F(T, T, x)$ per annum.

The forward annuity rate is set at time $t$ and is denoted by $F(t, T, x)$. The fair value of $F$ is

$$
\begin{aligned}
F(t, T, x) & =\frac{P(t, T) B(t, T, x)}{\sum_{s=T+1}^{\infty} P(t, s) B(t, s, x)}=\frac{\tilde{B}(t, T, x)}{X(t)} \\
\text { where } X(t) & =\sum_{s=T+1}^{\infty} \tilde{B}(t, s, x) .
\end{aligned}
$$

Note that $\tilde{B}(t, T, x)=P(t, T) B(t, T, x)$ and $X(t)$ are tradeable assets, and that $X(t)>0$ for all $t$.

Now there exists a measure $P_{X}$ equivalent to the real-world measure $P$ under which $V(t) / X(t)$ is a martingale for any tradeable asset with price process $V(t)$. It follows that $F(t, T, x)$ is such a martingale with stochastic differential equation

$$
d F(t, T, x)=F(t, T, x)\left[\gamma_{P}(t, T, x)^{\prime} d Z^{(X)}(t)+\gamma_{B}(t, T, x)^{\prime} d W^{(X)}(t)\right] .
$$


In this SDE:

- $Z^{(X)}(t)$ is a standard $n_{Z}$-dimensional Brownian motion under $P_{X}$ which drives the interest-rate process.

- $W^{(X)}(t)$ is a standard $n_{W^{-}}$-dimensional Brownian motion under $P_{X}$, independent of $Z^{(X)}(t)$, which drives developments in the mortality rates.

- $\gamma_{P}(t, T, x)$ and $\gamma_{B}(t, T, x)$ are $\mathcal{H}_{t}$-previsible volatility processes which indicate how the process $F(t, T, x)$ reacts to changes in the term structures of interest rates and mortality.

If we assume that $\gamma_{P}(t, T, x)$ and $\gamma_{B}(t, T, x)$ are deterministic processes then $F(s, T, x)$ for $t<s \leq T$ is log-normal under $P_{X}$ with

$$
\begin{aligned}
E_{P_{X}}\left[F(s, T, x) \mid \mathcal{H}_{t}\right] & =F(t, T, x) \\
\operatorname{Var}_{P_{X}}\left[\log F(s, T, x) \mid \mathcal{H}_{t}\right] & =\sigma_{F}(t, s, x)^{2} \\
& =\int_{t}^{s}\left(\left|\gamma_{P}(u, T, x)\right|^{2}+\left|\gamma_{B}(u, T, x)\right|^{2}\right) d u .
\end{aligned}
$$

We now consider the impact of option-like guarantees. Under a contract with an annuity guarantee, the annuity payable from $T$ is equal to

$$
\max \{K, F(T, T, x)\} \text {. }
$$

An annuity of $£ 1$ per annum from time $T$ has value $1 / F(T, T, x)$ so the value at $T$ of the guaranteed contract is, per survivor at $T$,

$$
\frac{\max \{K, F(T, T, x)\}}{F(T, T, x)}=1+G(T)
$$

where $G(T)=\max \{K-F(T, T, x), 0\} / F(T, T, x)$ represents the value at $T$ of the option component of the contract.

As noted above this is the value at $T$ per survivor at $T$. For policyholders who die before $T$, the contract expires worthless. Suppose then that we start with $N(0)$ policyholders at time 0 . In using a model which refers to individual policyholders we need to augment our filtration from $\mathcal{M}_{t}$ (which tells us about the development of $\mu(s, x+s)$ up to time $t)$ to $\mathcal{M}_{t}^{*}$ which provides additional information about the actual number of survivors out of the original $N(0)$.

Let $G(t)$ denote the value at $t$ of the option to policyholder $i$, assuming that he is one out of the $N(t)$ who are still alive at $t$. This option will pay off $G(T)$ at $T$ if the policyholder is still alive at $T$ or zero if he is dead at $T$ : equivalently the payoff at $T$ can be denoted by $G(T) I_{i}(T)$, where $I_{i}(s)=1$ if policyholder $i$ is still alive at time $s$ and zero otherwise. We can observe that $\sum_{i=1}^{N(0)} I_{i}(t) G(t)=$ $N(t) G(t)$ represents the price at $t$ of a tradeable asset that promises to pay $N(T) G(T)$ at $T$. The martingale property of $P_{X}$ then implies that 


$$
\begin{aligned}
\frac{N(t) G(t)}{X(t)} & =E_{P_{X}}\left[N(T) \frac{G(T)}{X(T)} \mid \mathcal{H}_{t}, N(t)\right] \\
& =E_{P_{X}}\left[N(T) \frac{(K-F(T, T, x))_{+}}{F(T, T, x)} \frac{1}{X(T)} \mid \mathcal{H}_{t}, N(t)\right] \\
& =E_{P_{X}}\left[N(T) \frac{(K-F(T, T, x))_{+}}{(\tilde{B}(T, T, x) / X(T))} \frac{1}{X(T)} \mid \mathcal{H}_{t}, N(t)\right] \\
& =E_{P_{X}}\left[N(T) \frac{(K-F(T, T, x))_{+}}{S(T, x)} \mid \mathcal{H}_{t}, N(t)\right] \\
& =E_{P_{X}}\left[E_{P_{X}}\left(N(T) \frac{(K-F(T, T, x))_{+}}{S(T, x)} \mid \mathcal{H}_{t}, N(t)\right) \mid \mathcal{H}_{t}, N(t)\right] \\
& =E_{P_{X}}\left[E_{P_{X}}\left(N(T) \mid \mathcal{H}_{t}, N(t)\right) \frac{(K-F(T, T, x))_{+}}{S(T, x)} \mid \mathcal{H}_{t}, N(t)\right] \\
& =E_{P_{X}}\left[\frac{N(t) S(T, x)}{S(t, x)} \frac{(K-F(T, T, x))_{+}}{S(T, x)} \mid \mathcal{H}_{t}, N(t)\right] \\
& =\frac{N(t)}{S(t, x)} E_{P_{X}}\left[(K-F(T, T, x))_{+} \mid \mathcal{H}_{t}\right] .
\end{aligned}
$$

In developing this identity, note that the equality (4.2) arises from the fact that $F(T, T, x)$ and $S(T, x)$ are $\mathcal{H}_{T}$-measurable random variables whereas under $P_{X}$ $N(T) \mid \mathcal{M}_{T}, N(t) \sim \operatorname{Binomial}(N(t), S(T, x) / S(t, x))$ (recalling Remark 2.1.2) leading to (4.3).

The lognormal property of $F(T, T, x)$ then implies that

$$
\begin{aligned}
G(t) & =\frac{X(t)}{S(t, x)}\left(K \Phi\left(-d_{2}\right)-F(t, T, x) \Phi\left(-d_{1}\right)\right) \\
\text { where } d_{1} & =\frac{\log F(t, T, x) / K+\frac{1}{2} \sigma_{F}(t, T, x)^{2}}{\sigma_{F}(t, T, x)} \\
d_{2} & =d_{1}-\sigma_{F}(t, T, x)
\end{aligned}
$$

where $\Phi(z)$ is the cumulative distribution function of the standard normal random variable.

\subsection{The SCOR market model: model 1}

It is clear from this development that the annuity market model is very useful for tackling the specific problem of how to price a guaranteed annuity option. 
However, for other problems, the model ceases to provide similarly elegant, analytical solutions. The same problem exists with the swap market model (Jamshidian, 1997): the Jamshidian approach provides an elegant solution for the price of a swaption, but is awkward to use in other situations. With interestrate modelling, the LIBOR market model (Brace, Gatarek and Musiela, 1997) tends to be the market model of choice for more general applications. In this section, we will develop the mortality equivalent of the LIBOR market model.

We will introduce this model by describing first a flexible form of annuity contract. Consider a cohort of identical policyholders who were aged $x$ at time 0 . Under this contract, for policyholder $i$ :

- Let $F_{i}\left(t^{-}\right)$be the policyholder's assets just before any transactions at time $t$.

- At time $t$, the policyholder draws an income of $P_{i}(t)$. This might be chosen at the discretion of the policyholder based upon information available up to time $t$, or it might be a prespecified proportion of $F_{i}\left(t^{-}\right)$.

- At time $t$, the insurer backing the contract undertakes to provide a return of $\tilde{s}(t, t+1, x)$ (which we call the survivor credit) at $t+1$ per unit at $t$ provided policyholder $i$ is still alive at that time. In return for this survivor credit, if the policyholder dies between $t$ and $t+1$, his assets will be retained by the insurer.

From the above description, the policyholder has no discretion over the choice of investment strategy. The funds that a surviving policyholder will have at time $(t+1)^{-}$are then

$$
F_{i}\left((t+1)^{-}\right)=\left(F_{i}\left(t^{-}\right)-P_{i}(t)\right)(1+\tilde{s}(t, t+1, x)) .
$$

The fair value for $\tilde{s}(t, t+1, x)$ requires that the value at $t$ of the payoff at $(t+1)$ to the policyholder is equal to $F_{i}\left(t^{-}\right)-P_{i}(t)$. Thus

$$
E_{Q}\left[\frac{C(t)}{C(t+1)} I_{i}(t+1)(1+\tilde{s}(t, t+1, x)) \mid \mathcal{H}_{t}, I_{i}(t)=1\right]=1
$$

where $I_{i}(t+1)=1$ if policyholder $i$ is still alive at $t+1$ and zero otherwise.

Now we can follow a similar argument to that used with the annuity market model to replace $I_{i}(t+1)$ in this expression with $S(t+1, x) / S(t, x)$. Noting also that $\tilde{s}(t, t+1, x)$ is set at time $t$, this implies that

$$
\begin{aligned}
\frac{\tilde{B}(t, t+1, x)}{\tilde{B}(t, t, x)}(1+\tilde{s}(t, t+1, x)) & =1 \\
\Rightarrow \tilde{s}(t, t+1, x) & =\frac{\tilde{B}(t, t, x)-\tilde{B}(t, t+1, x)}{\tilde{B}(t, t+1, x)} .
\end{aligned}
$$

Now the construction of the survivor credit, $\tilde{s}(t, t+1, x)$, is reminiscent of how LIBOR rates are calculated. Indeed, if we set mortality rates to zero then $\tilde{s}(t, t+1, x)$ is equal to the 12 -month LIBOR rate 


$$
L(t, t, t+1)=\frac{1-P(t, t+1)}{P(t, t+1)}
$$

(using the forward LIBOR notation $\left.L\left(t, T_{0}, T_{1}\right)=\left(P\left(t, T_{0}\right)-P\left(t, T_{1}\right)\right) / P\left(t, T_{1}\right)\right)$. For this reason we choose to refer to $\tilde{s}(t, t+1, x)$ as the Survivor Credit Offer Rate, or SCOR.

Now let us define the forward SCOR. At time $t$, a contract is entered into between policyholder $i$ (age $x+t$ at time $t$ ) and the insurer that specifies that the survivor credit payable between the future dates $T$ and $T+1$ will be $\tilde{s}(t, T$, $T+1, x)$. Under this contract:

- No payment is made at $t$.

- If policyholder $i$ is still alive at time $T$, he will pay $£ 1$ to the insurer at $T$.

- If policyholder $i$ is still alive at time $T+1$, he will receive $£ 1+\tilde{s}(t, T, T+1, x)$ from the insurer at $T+1$.

The value at $t$ to the policyholder of this transaction is

$$
(1+\tilde{s}(t, T, T+1, x)) \frac{\tilde{B}(t, T+1, x)}{B(t, t, x)}-\frac{\tilde{B}(t, T, x)}{B(t, t, x)} .
$$

The fair forward SCOR is thus

$$
\tilde{s}(t, T, T+1, x)=\frac{\tilde{B}(t, T, x)-\tilde{B}(t, T+1, x)}{\tilde{B}(t, T+1, x)} .
$$

Following the development of the LIBOR market model, we note that $\tilde{B}(t, T, x)-$ $\tilde{B}(t, T+1, x)$ and $\tilde{B}(t, T+1, x)$ for $t \leq T$ are both tradeable assets. Since $\tilde{B}(t, T+1, x)$ is also strictly positive we can use it as a numeraire. Thus there exists a measure, $P_{T+1, x}$ under which $U(t) / \tilde{B}(t, T+1, x)$ is a martingale for any tradeable asset with price process $U(t)$. Specifically $\tilde{s}(t, T, T+1, x)$ is a martingale under $P_{T+1, x}$.

Now positive interest and mortality imply that $0<\tilde{B}(t, T+1, x) / \tilde{B}(t, T, x)<1$. It follows therefore that $0<\tilde{s}(t, T, T+1, x)<\infty$, so we have the possibility to model $\tilde{s}(t, T, T+1, x)$ as a lognormal process. Thus we can write

$$
\begin{aligned}
d \tilde{s}(t, T, T+1, x)= & \tilde{s}(t, T, T+1, x)\left(\phi_{P}(t, T, T+1, x)^{\prime} d Z^{(T+1, x)}+\right. \\
& \left.\phi_{B}(t, T, T+1, x)^{\prime} d W^{(T+1, x)}\right)
\end{aligned}
$$

for suitable previsible volatility vectors $\phi_{P}$ and $\phi_{B}$ and where $Z^{(T+1, x)}(t)$ and $W^{(T+1, x)}(t)$ are Brownian motions under $P_{T+1, x}$.

Remark 4.3.1. It should be noted that while $0<\tilde{s}(t, T, T+1, x)<\infty$ does guarantee that $0<\tilde{B}(t, T+1, x) / \tilde{B}(t, T, x)<1$, it does not guarantee that 
$p_{Q}(t, T+1, x) / p_{Q}(t, T, x)<1$ : that is, there is a possibility that forward survival probabilities might exceed 1.

We will address this drawback in model 2 below. However, the particular parametrisation of the model may mean that the risk that this ratio exceeds 1 is very small. However, whatever the risk is, its consequences need to be carefully investigated.

Remark 4.3.2. If the volatility functions $\phi_{P}$ and $\phi_{B}$ are deterministic, $\tilde{s}(u, T$, $T+1, x)$ for $t<u \leq T$ has a log-normal distribution under $P_{T+1, x}$ with

$$
\begin{aligned}
& E_{P_{T+1, x}}\left[\tilde{s}(u, T, T+1, x) \mid \mathcal{H}_{t}\right]=\tilde{s}(t, T, T+1, x) \\
& \text { and } \\
& \operatorname{Var}_{P_{T+1, x}}\left[\log \tilde{s}(u, T, T+1, x) \mid \mathcal{H}_{t}\right]= \\
& \quad \int_{t}^{u}\left(\left|\phi_{P}(v, T, T+1, x)\right|^{2}+\left|\phi_{B}(v, T, T+1, x)\right|^{2}\right) d v .
\end{aligned}
$$

\subsection{The SCOR market model: model 2}

The SCOR market model 1 has several drawbacks including a restriction on the investment at $T$ to a fixed quantity and the possibility that forward survival probabilities exceed 1 (as in Remark 4.3.1). We now adapt this basic model to circumvent these problems. Consider, therefore, the following forward contract struck at time $t$ :

- At time $t$, no money exchanges hands.

- At time $T$, if the policyholder is still alive, he will pay $£ X(T)$ to the insurer. We allow $X(T)$ to be random but restrict it to be $\mathcal{F}_{T}$-measurable (that is, adapted to the interest-rate model dynamics).

- At time $T$, the policyholder can choose how his fund of $£ X(T)$ will be invested.

- At time $T+1$, if the policyholder is still alive, he will receive $£ X(T) R(T+1)$ / $R(T)(1+s(t, T, T+1, x))$ where $R(u)$ represents the price at $u$ of a non-mortality-linked tradeable asset (that is, $\mathcal{F}_{t}$-measurable) that reflects the policyholder's chosen previsible investment strategy. If the policyholder is not alive at $T+1$, then the funds are retained by the insurer.

Remark 4.4.1. For convenience, we will continue to refer to $s(t, T, T+1, x)$ as the forward Survivor Credit Offer Rate, although it should be stressed that the definition of this term is different from that under model 1.

Remark 4.4.2. The use of the SCOR in our modelling does not constrain us into looking only at contracts whose benefits are defined in terms of the SCOR. Rather, 
the model provides us with a means of modelling the development of mortality rates over time, since these can be easily backed out from the $s(t, T, T+1)$.

We now ask the question: what value of the forward SCOR, $s(t, T, T+1, x)$, ensures that this contract has zero value at time $t$ ? Let $I(u)$ be the indicator random variable that is equal to 1 if the policyholder is still alive at time $u$. Thus, for $u>t, \operatorname{Pr}_{Q}\left[I(u)=1 \mid I(t)=1, \mathcal{M}_{u}\right]=S(u, x) / S(t, x)$. For general $\tilde{S}=1+$ $s(t, T, T+1, x)$ the value at $t$ is

$$
\begin{gathered}
E_{Q}\left[\frac{C(t)}{C(T)} X(T)\left(\frac{C(T)}{C(T+1)} \frac{R(T+1)}{R(T)} \tilde{S} I(T+1)-I(T)\right) \mid \mathcal{H}_{t}, I(t)=1\right] \\
=\tilde{S} E_{Q}\left[\frac{C(t)}{C(T+1)} X(T) \frac{R(T+1)}{R(T)} \mid \mathcal{F}_{t}\right] E_{Q}\left[I(T+1) \mid \mathcal{M}_{t}, I(t)=1\right] \\
\quad-E_{Q}\left[\frac{C(t)}{C(T)} X(T) \mid \mathcal{F}_{t}\right] E_{Q}\left[I(T) \mid \mathcal{M}_{t}, I(t)=1\right] \\
=\tilde{S} E_{Q}\left[\frac{C(t)}{C(T)} X(T) \mid \mathcal{F}_{t}\right] p_{Q}(t, T+1, x) \\
\quad-E_{Q}\left[\frac{C(t)}{C(T)} X(T) \mid \mathcal{F}_{t}\right] p_{Q}(t, T, x) \quad \text { (recalling Remark 2.1. }
\end{gathered}
$$

which we require to be equal to zero to obtain the fair forward $\operatorname{SCOR}, s(t, T$, $T+1, x)$. This implies that

$$
\begin{aligned}
(1+s(t, T, T+1, x)) & p_{Q}(t, T+1, x)-p_{Q}(t, T, x)=0 \\
\Rightarrow s(t, T, T+1, x) & =\frac{p_{Q}(t, T, x)-p_{Q}(t, T+1, x)}{p_{Q}(t, T+1, x)} \\
& =\frac{B(t, T, x)-B(t, T+1, x)}{B(t, T+1, x)} .
\end{aligned}
$$

It follows that this revised SCOR market model has considerably wider applicability than the SCOR model 1. Specifically, the policyholder can adopt any $\mathcal{F}_{t}$-measurable investment strategy (but not strategies that depend, in addition, on changes in mortality). Furthermore, the amount of income that can be drawn from a policyholder's account can also be stochastic, provided it is an $\mathcal{F}_{t}$-measurable process.

Now we need to investigate whether or not the forward SCOR can be constructed out of tradeable assets and this indeed turns out to be the case. Specifically, let $R(t)$ be an $\mathcal{F}_{t}$-adapted process that represents the price at $t$ of a tradeable asset. Let $\tilde{D}(t, T, x)$ represent the price at $t$ for the investment and mortality-linked zero-coupon bond that pays $R(T) S(T, x)$ at $T$. Clearly $\tilde{D}(t, T, x)$ represents the price of a tradeable asset (one that could be replicated using the 
$P(t, T)$ and the $\tilde{B}(t, T, x))$. Furthermore, since $S(T, x)$ is independent of $\{Z(u)$ : $0<u \leq T\}$, we have

$$
\begin{aligned}
\tilde{D}(t, T, x) & =E_{Q}\left[\frac{C(t)}{C(T)} R(T) S(T, x) \mid \mathcal{H}_{t}\right] \\
& =E_{Q}\left[\frac{C(t)}{C(T)} R(T) \mid \mathcal{F}_{t}\right] E_{Q}\left[S(T, x) \mid \mathcal{M}_{t}\right] \\
& =R(t) B(t, T, x) .
\end{aligned}
$$

Hence

$$
\begin{aligned}
s(t, T, T+1, x) & =\frac{B(t, T, x)-B(t, T+1, x)}{B(t, T+1, x)} \\
& =\frac{R(t) B(t, T, x)-R(t) B(t, T+1, x)}{R(t) B(t, T+1, x)} \\
& =\frac{\tilde{D}(t, T, x)-\tilde{D}(t, T+1, x)}{\tilde{D}(t, T+1, x)} .
\end{aligned}
$$

As before there exists a measure $P_{T+1, x}^{D}$ under which the process $U(t) / \tilde{D}(t, T+1, x)$ is a martingale for all tradeable-asset price processes $U(t)$. Hence $s(t, T, T+1, x)$ is a martingale under $P_{T+1, x}^{D}$. Since we are constraining ourselves to models under which $B(t, T, x)$ is a decreasing function of $T$, we have $s(t, T, T+1, x)>0$. Hence the $\operatorname{SDE}$ for $s(t, T, T+1, x)$ can be written in the form

$$
d s(t, T, T+1, x)=s(t, T, T+1, x) \psi_{B}(t, T, T+1, x)^{\prime} d W^{(T+1, x)}
$$

for a suitable previsible volatility vector $\psi_{B}$ and $W^{(T+1, x)}(t)$ is a standard $n_{W^{-}}$ dimensional Brownian motion under $P_{T+1, x}^{D}$.

Remark 4.4.3. In contrast to model 1 , model 2 does constrain the $p_{Q}(t, T, T+1, x)$ to lie between 0 and 1 .

Remark 4.4.4. If the volatility function $\psi_{B}(t, T, T+1, x)$ is deterministic, $s(u, T, T+1, x)$ for $t<u \leq T$ has a log-normal distribution under $P_{T+1, x}^{D}$ with

$$
\begin{aligned}
E_{P_{T+1, x}^{D}}\left[s(u, T, T+1, x) \mid \mathcal{H}_{t}\right] & =s(t, T, T+1, x) \\
\text { and } \operatorname{Var}_{P_{T+1, x}^{D}}\left[\log s(u, T, T+1, x) \mid \mathcal{H}_{t}\right] & =\int_{t}^{u}\left|\psi_{B}(v, T, T+1, x)\right|^{2} d v .
\end{aligned}
$$

\subsubsection{Simulation}

Naturally we are interested in using the model to determine spot survival probabilities over a range of future dates $T$. So far equation (4.5) only tells us something 
about the dynamics of a single forward SCOR, $s(t, T, T+1, x)$. How do the dynamics of the $s(t, T, T+1, x)$ interact for different $T$ and how can they be simulated?

Application of the quotient rule for SDE's to equation (4.4) gives us

$$
\psi_{B}(t, T, T+1, x)=(V(t, T, x)-V(t, T+1, x)) \frac{(1+s(t, T, T+1, x))}{s(t, T, T+1, x)} .
$$

Now define a series of future dates $T_{k}=T_{0}+k$ for some $T_{0}>0$ and $k=1,2, \ldots$ Equation (4.6) can be rearranged to give

$$
V\left(t, T_{k}, x\right)-V\left(t, T_{k+1}, x\right)=\frac{s\left(t, T_{k}, T_{k+1}, x\right)}{\left(1+s\left(t, T_{k}, T_{k+1}, x\right)\right)} \psi_{B}\left(t, T_{k}, T_{k+1}, x\right) .
$$

Bearing in mind the relationship between the $W^{\left(T_{k}, x\right)}(t)$ and $\tilde{W}(t)$, for $l>k$ we have

$$
\begin{aligned}
d W^{\left(T_{l}, x\right)}(t) & =d \tilde{W}(t)-V\left(t, T_{l}, x\right) d t \\
d W^{\left(T_{k}, x\right)}(t) & =d \tilde{W}(t)-V\left(t, T_{k}, x\right) d t \\
\Rightarrow d W^{\left(T_{l}, x\right)}(t) & =d W^{\left(T_{k}, x\right)}(t)+\left(V\left(t, T_{k}, x\right)-V\left(t, T_{l}, x\right)\right) d t \\
& =d W^{\left(T_{k}, x\right)}(t)+\sum_{j=k+1}^{l}\left(V\left(t, T_{j-1}, x\right)-V\left(t, T_{j}, x\right)\right) d t \\
& =d W^{\left(T_{k}, x\right)}(t)+\sum_{j=k+1}^{l} \frac{s\left(t, T_{j-1}, T_{j}, x\right)}{1+s\left(t, T_{j-1}, T_{j}, x\right)} \psi_{B}\left(t, T_{j-1}, T_{j}, x\right) d t .
\end{aligned}
$$

by (4.7).

Thus, expressing the dynamics under $P_{T_{1}}^{D}$, we have

$$
d s\left(t, T_{0}, T_{1}, x\right)=s\left(t, T_{0}, T_{1}, x\right) \psi_{B}\left(t, T_{0}, T_{1}, x\right) d W^{\left(T_{1}, x\right)}(t)
$$

and for $k>1$

$$
\begin{aligned}
d s\left(t, T_{k-1}, T_{k}, x\right) & =s\left(t, T_{k-1}, T_{k}, x\right) \psi_{B}\left(t, T_{k-1}, T_{k}, x\right) \\
& \times\left(d W^{\left(T_{1}, x\right)}(t)+\sum_{j=2}^{k} \frac{s\left(t, T_{j-1}, T_{j}, x\right)}{1+s\left(t, T_{j-1}, T_{j}, x\right)} \psi_{B}\left(t, T_{j-1}, T_{j}, x\right) d t\right) .
\end{aligned}
$$

These equations can be used as the basis for simulation of the $s\left(t, T_{k-1}, T_{k}, x\right)$ and as a package it offers us a flexible and powerful toolkit for valuation of mortality-linked securities and for risk measurement. For the latter purpose, we would simulate under the real world measure $P$ by replacing $d W^{T_{1}}(t)$ by $d W(t)+\lambda(t) d t$ for a suitable process $\lambda(t)$. 


\subsection{The Perks-SCOR market model}

We now consider a specific version of the SCOR market model 2 by adapting a simple short-rate model proposed by Cairns, Blake and Dowd (2006) (CBD) for the age range 60 to 90 . CBD developed a model for the retrospective survival probability $p_{Q}(t+1, t, t+1, x)=1 /\left(1+\exp \left\{A_{1}(t+1)+A_{2}(t+1)(x+t)\right\}\right)$ (using the notation for forward survival probabilities in equation (2.7)). The parameters $A_{1}(t+1)$ and $A_{2}(t+1)$ are typically estimated in each year $(t, t+1]$ using mortality and population data for the same year without reference to data in preceeding years. This model for $p_{Q}(t, t+1, x)$ is a special case of what are known as Perks models (see, for example, Perks, 1932). The vector process $A(t)=$ $\left(A_{1}(t), A_{2}(t)\right)^{\prime}$ is modelled by CBD as a 2 -dimensional random walk with drift.

In the model we adopt here, we apply the same process, $A(t)$, to model oneyear spot survival probabilities rather than retrospective probabilities: that is,

$$
p_{Q}(t, t+1, x) \equiv p_{Q}(t, t, t+1, x)=\frac{1}{1+e^{A_{1}(t)+A_{2}(t)(x+t)}} .
$$

In the normal course of events, $p_{Q}(t, t+1, x)$ will be a very accurate predictor of $p_{Q}(t+1, t, t+1, x)$, so the change in concept used here does not undermine the simple model proposed by CBD.

As commented above, analysis of historical data suggest that a reasonable model for the discrete-time process $A(t)=\left(A_{1}(t), A_{2}(t)\right)^{\prime}$ is a random walk with drift: that is,

$$
A(t+1)=A(t)+\mu+C \Delta W(t+1)
$$

where $\mu$ is a $2 \times 1$ constant vector, $C=\left(c_{i j}\right)$ is a $2 \times 2$ constant matrix and $\Delta W(t+1)$ is a $2 \times 1$ vector of independent standard normal random variables.

Now we can easily write $A(t)$ as a continuous process:

$$
A(t)=A(0)+\mu t+C W(t)
$$

where $W(t)$ is a standard 2-dimensional Brownian motion. Thus $d A(t)=\mu d t+$ $C d W(t)$.

Returning to the SCOR market model, we are interested in modelling the future distribution of

$$
s(T, T, T+1, x)=\frac{1-p_{Q}(T, T+1, x)}{p_{Q}(T, T+1, x)}=\frac{q_{Q}(T, T+1, x)}{p_{Q}(T, T+1, x)} .
$$

Now the Perks model above implies that

$$
s(T, T, T+1, x)=e^{A_{1}(T)+A_{2}(T)(x+T)} .
$$

Now we require the forward SCOR $s(t, T, T+1, x)$ to be a martingale under $P_{T+1, x}^{D}$ : that is, 


$$
s(t, T, T+1, x)=\exp \left[\theta^{\prime}(A(t)+\mu(T-t))+\frac{1}{2} \theta^{\prime} C C^{\prime} \theta(T-t)\right]
$$

where $\theta=(1,(x+T))^{\prime}$. This implies that the volatility process for what we will now call the Perks-SCOR market model should be

$$
\begin{aligned}
\psi_{B}(t, T, T+1, x) & =v_{1}+v_{2}(x+T) \\
\text { where } v_{1} & =\left(\begin{array}{l}
c_{11} \\
c_{12}
\end{array}\right) \\
\text { and } v_{2} & =\left(\begin{array}{l}
c_{21} \\
c_{22}
\end{array}\right) .
\end{aligned}
$$

We then have the simple result that under $P_{T+1, x}^{D}, s(T, T, T+1, x)$ given $s(t, T$, $T+1, x)$ is log-normally distributed with

$$
\begin{gathered}
E_{P_{T+1, x}^{D}}\left[s(T, T, T+1, x) \mid \mathcal{M}_{t}\right]=s(t, T, T+1, x) \\
\text { and } \\
\operatorname{Var}_{P_{T+1, x}^{D}}\left[\log s(T, T, T+1, x) \mid \mathcal{M}_{t}\right]=\left[\left(c_{11}+c_{21}(x+T)\right)^{2}+\left(c_{12}+c_{22}(x+T)\right)^{2}\right](T-t) .
\end{gathered}
$$

Remark 4.5.1. As we have noted before, the differences between the Perks-SCOR market model and that of Cairns, Blake and Dowd (2006) are not great. The mechanisms underlying a market model will, however, mean that the drift processes for the two models are different.

\section{Conclusions}

We have presented here a number of theoretical frameworks that could be used for pricing many different types of mortality derivatives. More specifically, these frameworks demonstrate how an arbitrage-free (or risk-neutral) valuation methodology can be used to price a great variety of mortality-related instruments, from vanilla longevity bonds to exotic mortality derivatives. These frameworks build on those already established for conventional interest-rate derivatives, and therefore help to show how the existing literature on the valuation of interest-rate derivatives can be adapted to the valuation of derivatives involving mortality risk factors. They also provide a basis for the future development of specific stochastic mortality models, which can be developed within the frameworks offered here.

Our valuation methodology is of course the same, in principle, as that used to price derivatives based on other underlyings. As such, it is also open to exactly the same arguments over its merits. In particular, the use of arbitrage-free methods is always problematic if markets are incomplete, as is certainly the case with mortality derivatives markets. However, risk-neutral approaches provide 
natural benchmark valuations, and one can also argue heuristically that they will become easier to justify in any given context as markets become less incomplete over time. Naturally, we also recognize that many of the assumptions underpinning these frameworks (such as liquid, frictionless markets) do not hold in practice. Nevertheless, it is still the case that, in such imperfect markets, if prices evolve in the way suggested by these pricing frameworks, then the model will be arbitrage free. We are not assuming that the market must be complete, or that transactions costs must be zero or that assets are infinitely divisible, and so on.

Needless to say, many challenges remain for future work. These challenges centre around five main issues:

- Models: We need to investigate which models give adequate statistical descriptions of historical mortality data, and which models generate 'reasonable' mortality dynamics.

- The number of risk factors: We need to determine how many risk factors are needed to provide a satisfactory model of the mortality term structure. Is one risk factor adequate, or do we need to have two or more factors to accommodate imperfectly correlated mortality improvements at different ages? One can easily argue that multiple factors might be desirable because the factors that affect mortality (medical advances, outbreaks of disease, etc.) are likely to have different impacts on people of different ages.

- Basis risk vs. liquidity: As with other derivatives, there is the usual tradeoff between basis risk and liquidity. If we want to encourage the development of market liquidity, then we would encourage standardized mortality instruments trading on organized exchanges; however, such instruments will embody considerable basis risk, which will reduce their usefulness as hedge instruments. Conversely, the basis risk for the primary client associated with tailor-made (or OTC) instruments will be low, but at the cost of poor liquidity. However, there are also other factors to consider. For example, the trading of mortality derivatives can be encouraged by choosing 'good' reference populations for the mortality indices (e.g., a good reference population might one be typical of the population as a whole, or typical of the annuitant population). Furthermore, the market for mortality derivatives might be helped by the fact that mortality risks have low market beta, and the willingness of investors to buy up the Swiss Re mortality-related bond is certainly encouraging.

- Index specification and moral hazard: The choice of index also needs to take account of possible moral hazard. For example, can the index be manipulated by the issuer of a security or by investors? Issuers of mortality-linked securities can learn from past experience of cat bond issuance, where moral hazard is always present to some degree ${ }^{8}$. A carefully-chosen index will not

8 For a discussion of moral hazard as it relates to the issue of cat bonds, see, for example, the papers by Doherty (1997) and Doherty and Richter (2002). 
only increase liquidity, as discussed above, but it will also reduce moral hazard. A consequence, though, is that this reduction is typically accompanied by increased basis risk for those wishing to use the security for hedging. With cat bonds, one of the principal ways to reduce moral hazard is to link the payments to a non-company-specific index rather than to the ceding company's experience. The same arguments apply to mortality-related securities, although, perhaps, to a lesser degree: in other words, it is advisable that the survivor index defined in equation (2.1) is not calculated using the hedgers' own mortality experience.

- The measurement-publication lag: How do we allow for the time lag between the measurement date and the date when mortality rates for that date have been graduated and made public? For example, is there something that can be learned here from catastrophe derivatives, where information gradually emerges after a catastrophic event? Connected to this issue is the need to specify the contract in a way that minimises insider-trading moral hazard associated with time lags in the release of information. This is a critical issue, not least because some early attempts to introduce non-life-insurance-linked catastrophe derivatives failed on precisely this point. We note though, that the Swiss Re mortality-related bond was issued successfully, implying that investors must have been reassured on the issue of moral hazard.

In summary, mortality risks are an important new frontier in quantitative financial risk. They offer intellectual challenges to those who study them and serious financial benefits to those who trade them - and one would hope that they might also bring significant benefits to customers down the line.

\section{ACKNOWLEDGEMENTS}

The authors would like to thank both referees for their helpful comments on an earlier version of this paper. AC would like to thank David Wilkie for some invaluable discussions on the topic of mortality improvements and Vincent Vandier and Jean Berthon at AFPEN, Sam Cox, Kristian Miltersen and SveinArne Persson for some useful insights. We are also grateful to Iain Currie for providing the data for Figures 1.1 and 1.2 and to Owen Beelders for providing further details on the Swiss Re mortality bond.

Part of this work was carried out while AC was a visitor at the Isaac Newton Institute, Cambridge during the Developments in Quantitative Finance Programme, and also when AC, DB and KD were in receipt of the ESRC research grant RES-000-23-1036: Survivor Derivatives.

\section{REFERENCES}

Ahn, A.-H., Dittmar, R.F. and Gallant, A.R. (2002) Quadratic term structure models: theory and evidence. Review of Financial Studies, 15, 243-288. 
Artzner, P. and Delbaen, F. (1995) Default risk insurance and incomplete markets. Mathematical Finance, 5, 187-195.

Beelders, O. and Colarossi, D. (2004) Modelling mortality risk with extreme value theory: The case of Swiss Re's mortality-indexed bond. Global Association of Risk Professionals, 4 (July/August), 26-30.

BIFFIS, E. (2005) Affine processes for dynamic mortality and actuarial valuations. Insurance: Mathematics and Economics, 37, 443-468.

Biffis, E. and Millossovich, P. (2004) The fair value of guaranteed annuity options. Working paper, Bocconi University, Milan.

Biffis, E. and Millossovich, P. (2005) A bidimensional approach to mortality risk. Working paper, Bocconi University, Milan.

BLACK, F. and KARASINSKI, P. (1991) Bond and option pricing when short rates are log-normal, Financial Analysts Journal, July-Aug, 52-59.

Blake, D. and BurRows, W. (2001) Survivor bonds: Helping to hedge mortality risk. Journal of Risk and Insurance, 68, 339-348.

Blake, D., Cairns, A.J.G. and Dowd, K. (2003) Pensionmetrics II: Stochastic pension plan design during the distribution phase. Insurance: Mathematics and Economics, 33, 29-47.

Blake, D., Cairns, A.J.G. and Dowd, K. (2006) Living with mortality: longevity bonds and other mortality-linked securities. To appear in British Actuarial Journal.

Brace, A., Gatarek, D. and Musiela, M. (1997) The market model of interest-rate dynamics. Mathematical Finance, 7, 127-155.

Brigo, D. and Mercurio, F. (2001) Interest Rate Models: Theory and Practice. Springer, Berlin.

Brounns, N., Denuit, M. and Vermunt J.K. (2002) A Poisson log-bilinear regression approach to the construction of projected life tables. Insurance: Mathematics and Economics, 31, 373393.

CAIRnS, A.J.G. (2004a) A family of term-structure models for long-term risk management and derivative pricing. Mathematical Finance, 14, 415-444.

CAIRns, A.J.G. (2004b) Interest-Rate Models: An Introduction. Princeton University Press, Princeton.

CAIRns, A.J.G., Blake, D. and Dowd, K. (2006) A two-factor model for stochastic mortality with parameter uncertainty. To appear in the Journal of Risk and Insurance.

Cairns, A.J.G., Blake, D., Dawson, P. and Dowd, K. (2005) Pricing the risk on longevity bonds. Life and Pensions, October, 41-44.

CAirns, A.J.G. and Garcia Rosas, S.A. (2004) A family of term-structure models with stochastic volatility. In Proceedings of the 3rd World Congress of the Bachelier Society, Chicago, and Proceedings of the 35th International ASTIN Colloquium, Bergen.

CMI (1978) Proposed standard tables for life office pensioners and annuitants. Continuous Mortality Investigation Reports, 3, 1-30.

CMI (1999) Projection factors for mortality improvement. Continuous Mortality Investigation Reports, 17, 89-108.

Cox, S.H., Fairchild, J.R. and Pedersen, H.W. (2000) Economic aspects of securitization of risk. ASTIN Bulletin, 30, 157-193.

Cox, J., Ingersoll, J. and Ross, S. (1985) A theory of the term-structure of interest rates. Econometrica, 53, 385-408.

Cowley, A. and Cummins, J.D. (2005) Securitization of life insurance assets and liabilities. Journal of Risk and Insurance, 72, 193-226.

Currie I.D., Durban, M. and Eilers, P.H.C. (2004) Smoothing and forecasting mortality rates. Statistical Modelling, 4, 279-298.

DAHL, M. (2004) Stochastic mortality in life insurance: Market reserves and mortality-linked insurance contracts. Insurance: Mathematics and Economics, 35, 113-136.

Dahl, M. and Møller, T. (2005) Valuation and hedging of life insurance liabilities with systematic mortality risk. In Proceedings of the 15th International AFIR Colloquium, Zurich. (Available online at http://www.afir2005.ch)

DOHERTY, N.A. (1997) Innovations in managing catastrophe risk. Journal of Risk and Insurance, 64, 713-718.

Doherty, N.A. and Richter, A. (2002) Moral hazard, basis risk, and gap insurance. Journal of Risk and Insurance, 69, 9-24. 
Dowd, K., Blake, D., Cairns, A.J.G. and Dawson, P. (2006) Survivor swaps. Journal of Risk and Insurance, 73, 1-17.

DuffiE, D. and KAN, R. (1996) A yield-factor model of interest rates. Mathematical Finance, 6, 379-406.

Flesaker, B. and Hughston, L.P. (1996) Positive interest. Risk, 9(1), 46-49.

Forfar, D.O. and SMiTh, D.M. (1987) The changing shape of English life Tables. Transactions of the Faculty of Actuaries, 40, 98-134.

Froot, K.A. and Stein, J.C. (1998) Risk management, capital budgeting, and capital structure policy for financial institutions: an integrated approach. Journal of Financial Economics, 47, 55-82.

Heath, D., Jarrow, R. and Morton, A. (1992) Bond pricing and the term structure of interest rates: A new methodology for contingent claims valuation. Econometrica, 60, 77-105.

JAmes, J. and WebBer, N. (2000) Interest Rate Modelling. Wiley: Chichester.

JAMSHIDIAN, F. (1997) LIBOR and swap market models and measures. Finance and Stochastics, 1, 293-330.

Joint Mortality Investigation Committee (JMIC) (1974) Considerations affecting the preparation of standard tables of mortality. Journal of the Institute of Actuaries, 101, 135-201.

Karatzas, I. and Shreve, S.E. (1998) Methods of Mathematical Finance. Springer-Verlag: New York.

Lando, D. (2004) Credit Risk Modeling: Theory and Applications. Princeton University Press: Princeton.

LANe, M.N. (2000) Pricing risk transfer transactions. ASTIN Bulletin, 30, 259-293.

LEE, P.J. (2000a) A general framework for stochastic investigations of mortality and investment risks. Presented at the Wilkiefest, Heriot-Watt University, March 2000.

LeE, R. (2000b) The Lee-Carter method for forecasting mortality, with various extensions and applications. North American Actuarial Journal, 4, 80-93.

LEE, R.D. and CARTER, L.R. (1992) Modeling and forecasting U.S. mortality. Journal of the American Statistical Association, 87, 659-675.

Lin, Y. and Cox, S.H. (2005a) Securitization of mortality risks in life annuities. Journal of Risk and Insurance, 72, 227-252.

Lin, Y. and Cox, S.H. (2005b) A mortality securitization model. Working paper, Georgia State University.

LuCiAnO, E. and Vigna, E. (2005) Non mean reverting affine processes for stochastic mortality. In Proceedings of the 15th International AFIR Colloquium, Zurich. (Available online at http://www.afir2005.ch)

Macdonald, A.S., Cairns, A.J.G., Gwilt, P.L. and Miller, K.A. (1998) An international comparison of recent trends in population mortality. British Actuarial Journal, 4, 3-141.

Macdonald, A.S., Bartlett, D., Berman, C., Daykin, C., Grimshaw, D. and Savill, P. (2003) Mortality improvements and the cohort effect. CMI Working Papers 1 and 2; Presented to the Staple Inn Actuarial Society on 11 March 2003; 46 pages. (Available online at http:// www.sias.org.uk)

Marocco, P. and Pitacco, E. (1998) Longevity risk and life annuity reinsurance. In Transactions of the 26th International Congress of Actuaries, Birmingham, 6, 453-479.

Milevsky, M.A. and Promislow, S.D. (2001) Mortality derivatives and the option to annuitise. Insurance: Mathematics and Economics, 29, 299-318.

Miltersen, K.R. and Persson, S.-A. (2005) Is mortality dead? Stochastic forward force of mortality determined by no arbitrage. Working paper, University of Bergen.

Miltersen, K., Sandmann, K. and Sondermann, D. (1997) Closed-form solutions for term structure derivatives with log-normal interest rates. Journal of Finance, 52, 409-430.

Muermann, A. (2004) Catastrophe derivatives. In The Encyclopaedia of Actuarial Science, Volume 1, pages 231-236, Wiley, Chichester.

Olivier, P. and JefFery, T. (2004) Stochastic mortality models. Presentation to the Society of Actuaries of Ireland. (Available online at http://www.actuaries.ie/Resources/events_papers/ PastCalendarListing.htm)

Pelsser, A. (2003) Pricing and hedging guaranteed annuity options via static option replication. Insurance: Mathematics and Economics, 33, 283-296. 
Perks, W. (1932) On some experiments in the graduation of mortality statistics. Journal of the Institute of Actuaries, 63, 12-57.

PitAcCo, E. (2002) Longevity risk in living benefits. Presented at the third annual CeRP conference "Developing an Annuity Market in Europe", Turin.

Rebonato, R. (2002) Modern Pricing of Interest-Rate Derivatives. Princeton University Press: Princeton.

Renshaw, A.E. and Haberman, S. (2003) Lee-Carter mortality forecasting with age-specific enhancement. Insurance: Mathematics and Economics, 33, 255-272.

Rogers, L.C.G. (1997) The potential approach to the term-structure of interest rates and foreign exchange rates. Mathematical Finance, 7, 157-164.

RutKowski, M. (1997) A note on the Flesaker \& Hughston model of the term structure of interest rates. Applied Mathematical Finance, 4, 151-163.

Sснмоск, U. (1999) Estimating the value of the WINCAT coupons of the Wintherthur Insurance Convertible Bond: A study of the model risk. ASTIN Bulletin, 29, 101-163.

Schönbucher, P. (2003) Credit Derivatives Pricing Models: Models, Pricing and Implementation. Wiley: Chichester.

Smith, A.D. (2005) Stochastic mortality modelling. Presentation at the Workshop on the Interface between Quantitative Finance and Insurance, Edinburgh, April 2005. (Available online at www.icms.org.uk/meetings/2005/quantfinance/sci_prog.html)

VASICEK, O. (1977) An equilibrium characterisation of the term structure. Journal of Financial Economics, 5, 177-188.

WANG, S.S. (1996) Premium calculation by transforming the layer premium density. ASTIN Bulletin, 26, 71-92.

WANG, S.S. (2000) A class of distortion operations for pricing financial and insurance risks. Journal of Risk and Insurance, 67, 15-36.

WANG, S.S. (2002) A universal framework for pricing financial and insurance risks. ASTIN Bulletin, 32, 213-234.

Wang, S.S. (2003) Equilibrium pricing transforms: new results using Buhlmann's 1980 economic model. ASTIN Bulletin, 33, 57-73.

WiLLETS, R.C. (1999) Mortality in the next millenium. Presented to the Staple Inn Actuarial Society on 7 December 1999. (Available online at www.sias.org.uk.)

YANG, S. (2001) Reserving, pricing and hedging for guaranteed annuity options. PhD Thesis, Heriot-Watt University, Edinburgh.

YANG, S., and HuANG, H.C. (2005) Optimal contribution rate and asset allocation for defined contribution pension plan incorporating longevity risk. Working paper, Soochow University, Taiwan.

\section{APPENDICES}

\section{A. THE LeE AND CARTER MODEL FOR STOCHASTIC MORTALITY}

Lee and Carter (1992) investigate the dynamics of the observed central mortality rates $m(t, x)$ for integer $t$ and $x$. Their model breaks $m(t, x)$ down into a log-bilinear model

$$
\log m(t, x)=a(x)+b(x) k(t)
$$

with the translation and scaling constraints that $\sum_{x} b(x)=1$ and $\sum_{t=T_{0}}^{T_{1}} k(t)=0$. $a(x)$ and $b(x)$ are non-parametric functions without any smoothing applied or 
functional form. Stage 1 of the modelling process estimates the functions $a(x)$, $b(x)$ and $k(t)$ without reference to a dynamic model for $k(t)$. For a given functional form for $a(x)$ and $b(x), k(t)$ is estimated directly using the data for date $t$ in isolation and without any assumption about its dynamic form. This is repeated until we optimise the fit over $a(x)$ and $b(x)$ subject to the scaling constraints above. Stage 2 of the modelling process then fits an ARIMA process to the $k(t)$.

\section{B. THE LeE AND YANG MODEL FOR STOCHASTIC MORTALITY}

Lee (2000a) and Yang (2001) proposed the following model for stochastic mortality.

Suppose that a deterministic forecast of annual mortality rates is made at time 0 . Thus $\hat{q}(x, t)$ represents the probability (as estimated at time 0 ) that an individual aged $x$ at time $t$ will die before time $t+1$ for each integer $x$ and $t$. The actual mortality experience is modelled as

$$
\begin{aligned}
q(x, t) & =\hat{q}(x, t) \exp \left[X(t)-\frac{1}{2} \sigma_{Y}^{2}+\sigma_{Y} Z_{Y}(t)\right] \\
\text { where } X(t) & =X(t-1)-\frac{1}{2} \sigma_{X}^{2}+\sigma_{X} Z_{X}(t)
\end{aligned}
$$

and $Z_{X}(t)$ and $Z_{Y}(t)$ are mutually independent sequences of i.i.d. standard normal random variables.

It follows that the $X(t)$ models the stochastic trend in the development of the mortality curve while the $-\frac{1}{2} \sigma_{Y}^{2}+\sigma_{Y} Z_{Y}(t)$ models one-off environmental variations in mortality (such as a major flu epidemic). From the limited data available, Yang found that $\sigma_{Y}$ was not significantly different from 0 . In contrast, however, Lin and Cox (2005b), using a longer run of US population data and a slightly different model, found that mortality jumps of the type represented by $\sigma_{Y} Z_{Y}(t)$ above do form a significant part of the development of mortality over time.

Note that while $\hat{q}(x, t)$ is an unbiased estimate for $q(x, t)$, the use of $\hat{q}(x, t)$ will not produce, in general, unbiased estimates for other quantities: for example, the survivor index, $S(t, x)$.

\section{The Milevsky and Promislow model FOR STOCHASTIC MORTALITY}

Milevsky and Promislow (2001) model the force of mortality in the form $\mu(t, x)=$ $\xi_{0} \exp \left(\xi_{1} x+Y_{t}\right)$ where $Y_{t}$ is an Ornstein-Uhlenbeck process with SDE $d Y_{t}=$ $-\alpha Y_{t} d t+\sigma d W_{t}$. Essentially this is equivalent to a Gompertz model with a timevarying scaling factor. 


\title{
D. The DAHL MODEl For STOCHASTIC MORTALITY
}

Dahl (2004) models the process for $\mu(t, x+t)$ as follows

$$
d \mu(t, x+t)=\alpha^{\mu}(t, x, \mu(t, x+t)) d t+\sigma^{\mu}(t, x, \mu(t, x+t)) d \tilde{W}(t) .
$$

He finds that if the drift and volatility are of the form

$$
\begin{aligned}
\alpha^{\mu}(t, x, \mu(t, x+t)) & =\delta^{\alpha}(t, x) \mu(t, x+t)+\zeta^{\alpha}(t, x) \\
\text { and } \sigma^{\mu}(t, x, \mu(t, x+t)) & =\sqrt{\delta^{\sigma}(t, x) \mu(t, x+t)+\zeta^{\sigma}(t, x)}
\end{aligned}
$$

for some deterministic functions $\delta^{\alpha}(t, x), \delta^{\sigma}(t, x), \zeta^{\alpha}(t, x)$ and $\zeta^{\sigma}(t, x)$ then

$$
p_{Q}(t, T, x)=e^{A(t, T, x)-B(t, T, x) \mu(t, x+t)}
$$

where the deterministic functions $A(t, T, x)$ and $B(t, T, x)$ are derived from differential equations involving $\delta^{\alpha}(t, x), \delta^{\sigma}(t, x), \zeta^{\alpha}(t, x)$ and $\zeta^{\sigma}(t, x)$.

Dahl's affine model has been extended by Biffis (2005) who added a jump process into the SDE (D.1).

\author{
ANDREW J.G. CAIRNS \\ Department of Actuarial Mathematics and Statistics \\ School of Mathematical and Computer Sciences \\ Heriot-Watt University \\ Edinburgh, EH14 4AS \\ United Kingdom \\ E-mail: A.Cairns@ma.hw.ac.uk \\ David Blake \\ Pensions Institute \\ Cass Business School \\ 106 Bunhill Row \\ London, EC1 Y 8TZ \\ United Kingdom \\ Kevin Dowd \\ Centre for Risk \& Insurance Studies \\ Nottingham University Business School \\ Jubilee Campus \\ Nottingham, NG8 1 BB \\ United Kingdom
}

